Swarthmore College

Works

6-1-2018

\title{
The Combined Impact Of IgLON Family Proteins Lsamp And Neurotrimin On Developing Neurons And Behavioral Profiles In Mouse
}

K. Singh

K. Lilleväli

Scott F. Gilbert

Swarthmore College, sgilber1@swarthmore.edu
A. Bregin
J. Narvik

See next page for additional authors

Follow this and additional works at: https://works.swarthmore.edu/fac-biology

Part of the Biology Commons

Let us know how access to these works benefits you

\section{Recommended Citation}

K. Singh, K. Lilleväli, Scott F. Gilbert, A. Bregin, J. Narvik, M. Jayaram, M. Rahi, J. Innos, A. Kaasik, E. Vasar, and M.-A. Philips. (2018). "The Combined Impact Of IgLON Family Proteins Lsamp And Neurotrimin On Developing Neurons And Behavioral Profiles In Mouse". Brain Research Bulletin. Volume 140, 5-18. DOI: 10.1016/j.brainresbull.2018.03.013

https://works.swarthmore.edu/fac-biology/551

This work is brought to you for free by Swarthmore College Libraries' Works. It has been accepted for inclusion in Biology Faculty Works by an authorized administrator of Works. For more information, please contact myworks@swarthmore.edu. 
Authors

K. Singh, K. Lilleväli, Scott F. Gilbert, A. Bregin, J. Narvik, M. Jayaram, M. Rahi, J. Innos, A. Kaasik, E. Vasar, and M.-A. Philips 


\section{Accepted Manuscript}

Title: The combined impact of IgLON family proteins Lsamp and Neurotrimin on developing neurons and behavioral profiles in mouse

Authors: Katyayani Singh, Kersti Lilleväli, Scott F. Gilbert, Aleksandr Bregin, Jane Narvik, Mohan Jayaram, Märt Rahi,

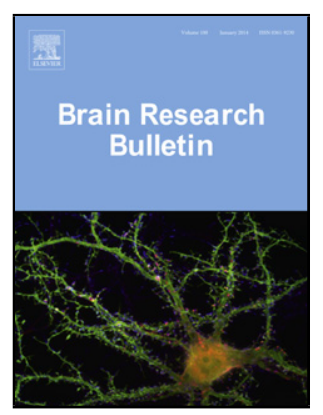

Jürgen Innos, Allen Kaasik, Eero Vasar, Mari-Anne Philips

PII:

DOI:

Reference:

S0361-9230(17)30720-7 https://doi.org/10.1016/j.brainresbull.2018.03.013

To appear in: $\quad$ Brain Research Bulletin

Received date: $\quad 1-12-2017$

Revised date: 26-2-2018

Accepted date: $\quad$ 23-3-2018

Please cite this article as: Katyayani Singh, Kersti Lilleväli, Scott F.Gilbert, Aleksandr Bregin, Jane Narvik, Mohan Jayaram, Märt Rahi, Jürgen Innos, Allen Kaasik, Eero Vasar, Mari-Anne Philips, The combined impact of IgLON family proteins Lsamp and Neurotrimin on developing neurons and behavioral profiles in mouse, Brain Research Bulletin https://doi.org/10.1016/j.brainresbull.2018.03.013

This is a PDF file of an unedited manuscript that has been accepted for publication. As a service to our customers we are providing this early version of the manuscript. The manuscript will undergo copyediting, typesetting, and review of the resulting proof before it is published in its final form. Please note that during the production process errors may be discovered which could affect the content, and all legal disclaimers that apply to the journal pertain. 


\section{The combined impact of IgLON family proteins Lsamp and Neurotrimin on developing neurons and behavioral profiles in mouse}

Katyayani Singh $^{\mathrm{a}, \mathrm{b}}$, Kersti Lilleväli ${ }^{\mathrm{a}, \mathrm{b}}$, Scott F Gilbert ${ }^{\mathrm{c}}$, Aleksandr Bregin ${ }^{\mathrm{a}, \mathrm{b}}$, Jane Narvik $^{\text {a,b }}$, Mohan Jayaram ${ }^{\mathrm{a}, \mathrm{b}}$, Märt Rahi ${ }^{\mathrm{d}}$, Jürgen Innos ${ }^{\mathrm{a}, \mathrm{b}}$, Allen Kaasik ${ }^{\mathrm{e}}$, Eero Vasar ${ }^{\mathrm{a}, \mathrm{b}}$, Mari-Anne Philips ${ }^{\mathrm{a}, \mathrm{b}}$ *

${ }^{a}$ Department of Physiology, Institute of Biomedicine and Translational Medicine, University of Tartu, 19 Ravila Street, 50411 Tartu, Estonia

${ }^{b}$ Centre of Excellence in Genomics and Translational Medicine, University of Tartu, 19 Ravila Street, 50411 Tartu, Estonia

${ }^{c}$ Department of Biology, Swarthmore College, Swarthmore, PA, USAd

${ }^{d}$ Institute of Agricultural and Environmental Sciences, Estonian University of Life Sciences, Fr.R. Kreutzwaldi 5 51014Tartu, Estonia

${ }^{e}$ Department of Pharmacology, Institute of Biomedicine and Translational Medicine, University of Tartu, 19 Ravila Street, 50411 Tartu, Estonia

* Correspondence: marianne.philips@ut.ee

\section{Graphical abstract}

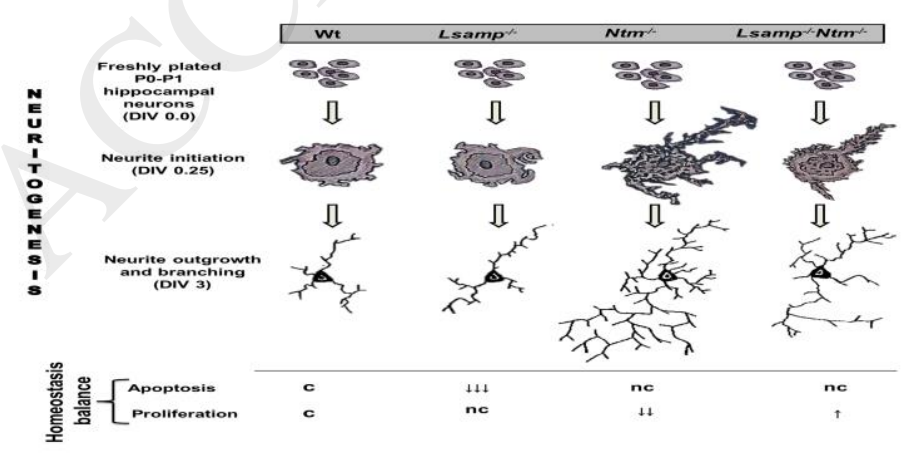




\title{
Highlights
}

- Combined Lsamp and/or Ntm mouse models showed interactions between IgLONs

- The initial phenotype on $\mathrm{Lsamp}^{-/-} \mathrm{Ntm}^{-/}$double-deficient mice is provided

- Lsamp and Ntm have combined impact on neuronal development and behaviour

- IgLONs can regulate neurite sprouting independent of adhesion between the cells

\begin{abstract}
Cell surface neural adhesion proteins are critical components in the complex orchestration of cell proliferation, apoptosis, and neuritogenesis essential for proper brain construction and behavior. We focused on the impact of two plasticity-associated IgLON family neural adhesion molecules, Neurotrimin (Ntm) and Limbic system associated membrane protein (Lsamp), on mouse behavior and its underlying neural development. Phenotyping neurons derived from the hippocampi of $\mathrm{Lsamp}^{-/}, \mathrm{Ntm}^{-/-}$and $\mathrm{Lsamp}^{-/-} \mathrm{Ntm}^{-/-}$mice was performed in parallel with behavioral testing.

While the anatomy of mutant brains revealed no gross changes, the $\mathrm{Ntm}^{-/-}$hippocampal neurons exhibited premature sprouting of neurites and manifested accelerated neurite elongation and branching. We propose that Ntm exerts an inhibitory impact on neurite outgrowth, whereas Lsamp appears to be an enhancer of the said process as premature neuritogenesis in $\mathrm{Ntm}^{-1}$ neurons is apparent only in the presence of Lsamp. We also show interplay between Lsamp and $\mathrm{Ntm}$ in regulating tissue homeostasis: the impact of $\mathrm{Ntm}$ on cellular proliferation was dependent on Lsamp, and Lsamp appeared to be a positive regulator of apoptosis in the presence of Ntm. Behavioral phenotyping indicated test-specific interactions between Lsamp and Ntm. The phenotypes of single mutant lines, such as reduced swimming speed in Morris water maze and increased activity in the elevated plus maze, were magnified in $\mathrm{Lsamp}^{-/-} \mathrm{Ntm}^{-/-}$ mice.
\end{abstract}

Altogether, evidence both from behavioral experiments and cultured hippocampal cells show combined and differential interactions between $\mathrm{Ntm}$ and Lsamp in the formation of hippocampal circuits and behavioral profiles. We demonstrate that mutual interactions between IgLON molecules regulate the initiation of neurite sprouting at very early ages, and even cellautonomously, independent of their regulation of cell-cell adhesion. 
Keywords (6): Adhesion molecules, Hippocampus, IgLON, Lsamp, Ntm, Neuritogenesis

\section{Introduction}

The initiation and maintenance of robust functional neuronal circuits depends on the delicate regulation of cellular proliferation, apoptosis, and the establishment of precise neuronal connections. The outgrowth and elongation of neurites at the appropriate times and in the correct directions provide the basis of functional neural connectivity (Silva and Dotti, 2002), and thus, cognitive and behavioral functions of the brain. Structural alterations during neuritogenesis are associated with abnormal neural circuit formation in psychiatric disorders such as autism spectrum disorders (Bakos et al., 2015) and schizophrenia (Lang et al., 2014). Disrupted functional brain connectivity, reflecting impairment of the integrity of white matter fiber tracts, has been found both in the patients with schizophrenia or bipolar disorder (Argyelan et al., 2014; Rashid et al., 2014; Li et al., 2017). Cell membrane molecules, such as the IgLON proteins, are critical for the formation of correct interactions between neural cells (Tan et al., 2017). The IgLON superfamily of cell adhesion molecules (CAMs) are the most abundantly expressed GPI-anchored neural cell surface glycoproteins (Salzer et al., 1996) in the neural cell membrane, and these proteins include Lsamp (limbic system associated membrane protein), Ntm (neurotrimin), Opcml (opioid-binding cell adhesion molecule), Kilon / Negr1 (neuronal growth regulator 1) and IgLON5 (Vanaveski et al., 2017). In the brain, Lsamp, Negr1 and Opcml are expressed both in neurons and oligodendrocytes except for NTM, which has been found to be enriched specifically in neurons (Sharma et al., 2015). IgLONs facilitate the assembling of the tuned groups of neurons during the formation of neuronal circuits (Kolodkin and Tessier-Lavigne, 2011; Schmidt et al., 2014). In particular, IgLONs have been shown to promote growth cone migration, axon target guidance, synapse formation, and dendritic tree formation, throughout development and adulthood (Miyata et al., 2003; Hashimoto et al., 2009; Yamada et al., 2007; Akeel et al., 2011; Pischedda et al., 2014; Sanz et al., 2017; Singh et al., 2018). Several studies have shown functional alterations of IgLON family members in several malignancies both in the brain and outside central nervous system (Chen et al., 2003; Ntougkos et al., 2005; Minhas et al., 2013; Kim et al., 2014), suggesting further functions in cellular homeostasis.

The number of potential interactions between IgLONs is extremely high due to their ability to form homophilic and heterophilic inter-family associations both in cis and trans orientations 
on the cell surface. In the current study we investigated the single and combined effects of two IgLONs, Lsamp and Ntm, on hippocampal development and mouse behaviors. On the plane of the neuronal membrane, heterophilic cis-interaction between Lsamp and Ntm has been shown to be one of the most likely IgLON combinations. Likewise the affinity between Lsamp and $\mathrm{Ntm}$ has been shown to be the highest when pairs of IgLONs were compared for their potential to form trans-interactions between neurons (Reed et al., 2004). Behavioral studies in Lsamp ${ }^{-/-}$ mice indicate the significance of Lsamp in the regulation of complex emotional and social behavior (Innos et al., 2011, 2012, 2013; Philips et al., 2015) whereas $\mathrm{Ntm}^{-/-}$mice exhibit deficit in emotional learning (Mazitov et al., 2017). Lsamp has been shown to be implicated in hippocampal plasticity and adaptation in changing environments (Qui et al., 2010; Heinla et al., 2015). Polymorphisms and expressional alterations in the Lsamp gene in humans have been linked to a spectrum of neuropsychiatric disorders (Must et al., 2008; Behan et al., 2009; Koido et al., 2012, 2014) whereas polymorphisms in the Ntm gene have been found to be associated with cognitive functions (Liu et al., 2007) and intelligence (Pan et al., 2010) and expressional alterations in the dorsolateral prefrontal cortex have been found in schizophrenic brains (Karis et al., 2018).

On the cellular level, IgLON cell adhesion molecules have been shown to modify specific neuronal projections that are relevant in modulating behavioral reactions. Ntm has been shown to participate in directing the thalamocortical and pontocerebellar projections (Struyk et al., 1995; Chen et al., 2001). Lsamp is involved in the establishment of thalamic, septo- and intrahippocampal circuits (Keller et al., 1989; Pimenta et al., 1995; Mann et al., 1998); and it is critical in the fasciculation of dopaminergic afferents from the midbrain to lateral habenula (Schmidt et al., 2014).

The neuroanatomical distribution of two IgLON members, Lsamp and Ntm, is highly heterogeneous throughout the brain, and it has been proposed that they are expressed by distinct complementary subpopulations of neurons with co-expression at a few sites (Philips et al., 2015; Gil et al., 2002). The brain areas expressing both Lsamp and Ntm include sensory and sensory-motor cortex, entorhinal cortex, hippocampus, amygdala, thalamus (ventral posteromedial, lateral geniculate nucleus and lateral dorsal nuclei), pyriform cortex, cerebellum, brain stem nuclei, spinal cord and dorsal root ganglia, (Philips et al., 2015; Struyk et al., 1995; Gil et al., 2002). In cultured hippocampal neurons, the co-expression of Lsamp and Ntm has been shown at the level of single neurons (Gil et al., 2002). Despite overlapping 
expression distribution and high affinity, Ntm and Lsamp have been shown to have differential effects on behavior and antagonistic functions in several cell culture studies (Hashimoto et al., 2009; Ntougkos et al., 2005; Mazitov et al., 2017). Therefore, the IgLON CAMs Ntm and Lsamp are an attractive couple to study for their distinct and combined abilities to affect neuronal function and the corresponding behavioral correlates.

To decipher the interaction between Lsamp and Ntm at different functional levels of the central nervous system, we investigated the neuronal development, gross anatomy of the brain and behavioral profile in Lsamp-deficient, $\mathrm{Ntm}$-deficient and Lsamp/Ntm double-deficient mice. As the only clearly overlapping change in phenotype for both $\mathrm{Lsamp}^{-/}$and $\mathrm{Ntm}^{-/}$animals has been found to be decreased sensitivity to amphetamine (Innos et al. 2013; Mazitov et al. 2017), the sensitivity to amphetamine in $\mathrm{Lsamp}^{-/} \mathrm{Ntm}^{-/}$mice was also tested in the current study. The process of neuritogenesis has been well-characterised in cultured hippocampal neurons (Craig et al., 1994), which were used in the current study to uncover the IgLON-associated morphological changes during development. The current study is the first to describe the early neuritogenesis and cellular homeostasis of $\mathrm{Lsamp}^{-/}, \mathrm{Ntm}^{-/}$and $\mathrm{Lsamp}^{-/-} \mathrm{Ntm}^{-/-}$hippocampal neurons. Behavioral phenotyping of mice with the same set of genotypes enabled us to gain insight concerning how the alterations in neuronal morphology become manifest at the behavioral level. 


\section{Materials and Methods}

\subsection{Experimental Animals}

The generation of Lsamp-deficient $\left(\right.$ Lsamp $\left.^{-/-}\right)$mice with a $L a c Z$ transgene has been described by Innos et al., 2011. Briefly, exon 1b of the murine Lsamp gene was replaced by an in-frame NLS-LacZ-NEO cassette resulting in the disruption of all functional Lsamp transcripts. Ntm gene heterozygous mutant strain (032496-UCD B6;129S5-Ntm ${ }^{\text {tmlLex }} / \mathrm{Mmucd}$ ) was obtained from the Mutant Mouse Regional Resource Centre at UC Davis (https://www.mmrrc.org/catalog/sds.php?mmrrc_id=32496) as described in Mazitov et al., 2017. Briefly, the strategy for the creation of $\mathrm{Ntm}$-deficient $\left(\mathrm{Ntm}^{-/-}\right)$mice was analogous to that of Lsamp-deficient mice, as exon $1 \mathrm{~b}$ was deleted, leading to the disruption of all functional Ntm transcripts.

We generated double heterozygous mice for Lsamp and Ntm by crossing Lsamp ${ }^{-/}$and $\mathrm{Ntm}^{-/-}$mice in F2 strain background [(129S5/SvEvBrd $\times$C57BL/6) $\times(129 \mathrm{~S} 5 / \mathrm{SvEvBrd} \times$ C57BL/6)]. Further crossing of the obtained double heterozygous mice $\left(\mathrm{Lsamp}^{+/-} \mathrm{Ntm}^{+/-}\right)$gave us the entire spectrum of genotypes (including $\mathrm{Lsamp}^{+/+} \mathrm{Ntm}^{+/+}, \mathrm{Lsamp}^{+/+} \mathrm{Ntm}^{-/-}, \mathrm{Lsamp}^{-/-} \mathrm{Ntm}^{+/+}$, $\mathrm{Lsamp}^{-/-} \mathrm{Ntm}^{-/-}$) that were used in the behavioral studies. For primary culture, breeding was done separately for all of the genotypes obtained from the above crossings in order to get a sufficient number of new-born pups with particular genotypes and comparable background. The behavioral experiments were performed with male mice $2-4$ months of age. Mice were grouphoused in standard laboratory cages measuring $42.5(\mathrm{~L}) \times 26.6(\mathrm{~W}) \times 15.5(\mathrm{H}) \mathrm{cm}, 6-8$ animals per cage in the animal colony at $22 \pm 1^{\circ} \mathrm{C}$, under a 12:12 h light/dark cycle (lights off at 19:00 h). A $2 \mathrm{~cm}$ layer of aspen bedding (Tapvei, Estonia) and 0.51 of aspen nesting material (Tapvei, Estonia) was used in each cage and changed every week. No other enrichment was used besides nesting material. Tap water and food pellets (R70, Lactamin AB, Sweden) were available $a d$ libitum. Breeding and housing of the mice was conducted at the animal facility of the Institute of Biomedicine and Translational Medicine, University of Tartu, Estonia. All animal procedures in this study were performed in accordance with the European Communities Directive (2010/63/EU) and permit (No. 29, April 28, 2014) from the Estonian National Board of Animal Experiments. 


\subsection{Primary hippocampal neuronal culture}

\subsubsection{Hippocampal neuronal culture stages followed in the current study}

Mouse hippocampal culture was classified into six different stages of development (Baj et al., 2014). At the molecular level, neuritogenesis involves the growth and orientation of cytoskeletal microtubules along actin filament (F-actin) bundles to initiate the growing neurites (Flynn, 2013). The stage 1 was defined as neurite initiation stage, when hippocampal cells protrude F-actin-rich lamellopodia veils tapered to form filopodia (Days in-vitro; DIV 0.25, 6 $\mathrm{h}$ post plating). The expanding filopodia allow the entry of microtubules to form minor neurite processes (Stage 2; DIV 0.5). Stage 3 (DIV 1-3) defines the outgrowth of primary dendrites and axon specification. Further Stages 4-6 involve progressive dendritic outgrowth and axonal stabilization, synaptogenesis and maturation to form functional neural circuits.

In order to study the earliest morphological changes during hippocampal neuronal development, we chose two stages of neuritogenesis: (1) the neurite initiation stage, where we investigated the dynamics of cytoskeleton rearrangement and quantified the redistribution of neuronal cytoskeletal actin using phalloidin, an F-actin-binding compound (Zhang et al., 2016). Microtubule-associated protein (MAP2) which interacts with F-actin during neurite initiation (Roger et al., 2004) was used as a neuronal cell marker. (2) At Stage 3 (DIV3), we labelled hippocampal neurons with neuron-specific pAAV-hSyn-RFP expression plasmid and scored for the following morphological characteristics: the number, length and branches of neurites. We also used Stage 3 hippocampal primary culture to elucidate the rate of apoptosis and proliferation during in-vitro development.

\subsubsection{Culture preparation}

Dissociated hippocampal primary neuronal cultures were prepared from whole hippocampi of postnatal 0-1 day old mouse pups. The brains were removed, chilled with frozen phosphate buffered saline (PBS), and the hippocampi were micro-dissected under sterile conditions according to Chatterjee et al., 2014. The hippocampi were digested with papain/DNase (20 $\mathrm{U} / \mathrm{ml}$ ) solution for $20 \mathrm{~min}$ at $37^{\circ} \mathrm{C}$ periodic stirring. The papain solution was aspirated and the hippocampal pieces were triturated with $1 \mathrm{ml}$ of culture medium. Following centrifugation at $1200 \mathrm{rpm}$ for $2 \mathrm{~min}$ the cells were suspended in culture media consisting Dulbecco's modified Eagle's medium F12 HAM (D8437) supplemented with N1 (N6530), 10\% fetal bovine serum (F4135) and antibiotic antimycotic solution (A5955). Approximately, 20,000-50,000 
dissociated neuronal cells were plated and grown onto $35 \mathrm{~mm}$ glass bottom dishes (glass surface diameter $14 \mathrm{~mm}$; MatTek, Ashland, MA) precoated with poly-D lysine $(0.1 \mathrm{mg} / \mathrm{ml})$. All the cultures were grown in a humidified $5 \% \mathrm{CO}_{2}$ incubator at $37^{\circ} \mathrm{C}$. Chemicals, culture media and supplements were obtained from Sigma-Aldrich (USA).

\subsubsection{Immunofluorescent staining and image analysis}

Primary hippocampal neurons grown on glass-bottom dishes were briefly washed twice with cold PBS, and fixed with 4\% PFA for 5-10 min at RT. After fixation, the neurons were washed with cold PBS $(3 \times 5 \mathrm{~min})$, permeabilized with $0.1 \%$ Triton X-100 in PBS for $10 \mathrm{~min}$, rinsed three times by using PBS and blocked with $1 \%$ BSA in PBS for $1 \mathrm{~h}$ at RT. The neurons were incubated with primary antibodies in $1 \% \mathrm{BSA} / \mathrm{PBS}$ in a humidified chamber overnight at $4{ }^{\circ} \mathrm{C}$; and the primary antibodies used in these studies included guinea pig anti-MAP2 (1:500; Synaptic Systems, 188004), rat anti-phospho-histone-H3 (PHH3) (1:500; Sigma Aldrich, H9908), guinea pig anti-doublecortin (DCX) (1:300; EMD-Millipore, AB2253), and rabbit anti-synapsin-1 (1:1000; EMD-Millipore, AB1543P). This was followed by incubation with secondary antibodies in 1\% BSA/PBS in a light-proof container (1-2 $\mathrm{h}$ at RT). Secondary antibodies used were fluorescein (FITC) donkey anti-guinea pig (1:1000; Jackson, 706-095148), rhodamine (TRITC) donkey anti-guinea pig (1:1000; Jackson, 706-025-148), Alexa Fluor 488 donkey anti-mouse (1:1000; Jackson, 715-545-150), Alexa Fluor 488 goat anti-rat (1:2500; Molecular Probes, ab 150157), and FITC-conjugated donkey anti-rabbit (1:2500; Jackson, 711-095-152). Phalloidin-TRITC (Sigma Aldrich) was diluted 1:50 in PBS and incubated for $30 \mathrm{~min}$ at RT to selectively stain F-actin. Finally, cells were washed $(3 \times 20 \mathrm{~min}$ in PBS), stained with $0.1 \mu \mathrm{g} / \mathrm{ml}$ DAPI for 2-5 min, washed in PBS and mounted with Vectasheild (Vector Labs).

Immunostaining was captured by an LSM 510 META Zeiss confocal microscope equipped with x 63/1.4 oil immersion objective. Acquired images were processed using LSM image browser (http://www.zeiss.co.uk/) and Photoshop CS4 Extended software (Adobe Systems Inc.). For Phalloidin quantification, Imaris 8.1 (Bitplane) was used to build 3D reconstructions from confocal $\mathrm{z}$ stacks of phalloidin-stained F-actin in the neurons using the "isosurface rendering" function of Imaris. Isosurface was created and the same threshold was set and used for the intensity measurement. These isosurface renderings were used to make quantitative measurements on the volume and surface fluorescent intensity. 


\subsubsection{Transfection}

Primary hippocampal neurons were transiently transfected on DIV 2 with plasmid pAAVhSyn-RFP, using Lipofectamine 2000 according to the instructions of the manufacturer (Invitrogen), (The pAAV-hSyn-RFP was a kind gift from Edward Callaway; Addgene plasmid

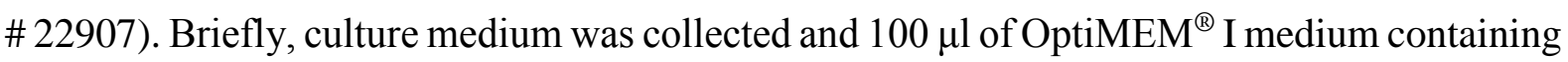
2\% Lipofectamine 2000 with $1 \mu \mathrm{g}$ of plasmid DNA was added directly to the cells grown on the glass bottom dishes and incubated for $3-4 \mathrm{~h}$ in a humidified $5 \% \mathrm{CO}_{2}$ incubator at $37^{\circ} \mathrm{C}$. The neurons were replenished with collected culture media and maintained for another $24 \mathrm{~h}$ to enable the expression of the transfected DNA. All the media and supplements and reagents were obtained from Invitrogen (Carlsbad, CA, USA).

\subsubsection{Neuronal imaging and neurite analysis}

The transfection of the pAAV-hSyn-RFP expression plasmid resulted in intense labelling of the entire neurite arbours of individual neurons at DIV3. Since a small number of individual neurons $(\sim 15-20 \%)$ were transfected in each culture, we were able to follow the primary neurite processes of separately labelled neurons. During the image acquisition at DIV3, the neurons were maintained in sterile PBS solution. Neurons selected for analysis were imaged using an Olympus IX70 inverted microscope equipped with WLSM Plan Apo ×20/1.25 objective and Olympus DP70 CCD camera. For neurite analysis, 25-30 transfected neurons were randomly captured from each experimental dish of 3-4 independent replication experiments. For the tracing of neurite number or neurite length per neuron, the total number or total length of projections arising from the cell soma was considered, while branching was determined on the basis of total number of nodes and ends according to Scholl analysis. The morphometric analysis was performed using computer-aided neuronal tracing software (Neurolucida Software; Version 11 64-bit, MBF Bioscience), and Scholl analysis was performed with Neuroexplorer software (MicroBrightField).

\subsubsection{Apoptosis Assay}

The primary hippocampal neuronal cultures were fixed at DIV3 with 4\% PFA for 5 min at RT, and TUNEL staining was performed using Neuro TACS ${ }^{\mathrm{TM}}$ II in situ apoptosis detection kit (Trevigen, Inc., Gaithersburg, MD) according to the manufacturer's instructions. Briefly, the fixed neurons were permeabilized with proteinase K (1:100 dilutions). Neurons were then incubated with TdT and biotin-labeled dUTP at $37^{\circ} \mathrm{C}$ for $1 \mathrm{~h}$. After being washed in PBS, the 
neurons were incubated with streptavidin-conjugated horseradish peroxidase, and visualized with diaminobenzidine and counterstained with Blue Counterstain (R\&D Systems, Minneapolis, MN). The counterstain allowed the visualization of all TUNEL-positive nuclei as a dark brown colour, while normal cells appeared as light tan. Neurons were visualized using an Olympus IX70 inverted microscope with $\times 20 / 1.25$ objective. The number of TUNELpositive cells relative to the total number of cells was determined from 10 randomly selected fields from the culture dish from three independent experiments.

\subsubsection{Scanning electron microscopy}

Primary neuronal cultures at DIV 0.25 (6 h post seeding) grown on poly-D-lysine-coated 12 $\mathrm{mm}$ glass coverslips were gently rinsed twice in pre-warmed $\left(37^{\circ} \mathrm{C}\right) 0.1 \mathrm{M}$ phosphate buffer $(\mathrm{pH}$ 7.3). The cultures were fixed with $2.5 \%$ glutaraldehyde in the same buffer for $30 \mathrm{~min}$ at RT. The samples were then rinsed in $0.1 \mathrm{M}$ phosphate buffer and post fixed for $30 \mathrm{~min}$ at RT in $1 \% \mathrm{OsO}_{4}$ in $0.1 \mathrm{M}$ phosphate buffer. Dehydration was carried out through series of ethanol solutions each for $5 \mathrm{~min}$ from $30 \%$ prepared in $0.1 \mathrm{M}$ phosphate buffer following $40 \%, 50 \%$, $70 \%$ and $90 \%$ in Milli-Q water. The samples were washed once with $99.8 \%$ ethanol and stored in $99.8 \%$ ethanol at $-20^{\circ} \mathrm{C}$ until critical-point-dried using liquid $\mathrm{CO}_{2}$. The samples were then sputter-coated with gold and viewed under scanning electron microscopy (SEM) Zeiss EVO MA-15.

\subsection{8. qRT-PCR analysis}

Total cell RNA was extracted from Wt control primary hippocampal cultures at DIV3 and from P3 Wt mouse hippocampi by using TRIZOL reagent (Invitrogen, USA) according to the manufacturer's protocol. Single-strand cDNA was synthesized using Random Hexamers (Applied Biosystems) and SuperScriptTM III Reverse Transcriptase (Invitrogen, USA). Quantitative TaqMan Assay with FAM-BHQ-probe was designed for the detection of Lsamp $1 a / 1 b$ and $N t m ~ l a / 1 b$ specific transcript by two-step RT-qPCR (qPCR) according to Vanaveski et al., 2017. TaqMan ${ }^{\circledR}$ Universal PCR Master Mix was used in the ABI Prism 7900HT Sequence Detection System (Applied Biosystems, USA). Reactions were carried out in $10 \mu 1$ reaction volumes in four replicates. 


\subsection{Histological analysis:}

\subsubsection{Nissl staining}

Brains from Wt, $\mathrm{Lsamp}^{-/}, \mathrm{Ntm}^{-/}$and $\mathrm{Lsamp}^{-/} \mathrm{Ntm}^{-/-}$adult mice were dissected and fixed overnight in $4 \%$ paraformaldehyde (PFA), and subsequently sectioned at $50 \mu \mathrm{m}$. Free floating sections were stained with $0.5 \%$ Cresyl violet for 7-10 min. The sections were washed with $\mathrm{dH}_{2} \mathrm{O}$, dehydrated with graded alcohols, cleared with xylene, mounted on glass slides using Pertex and imaged under brightfield microscopy.

\subsubsection{Neurofilament immunostaining}

The standard peroxidase anti-peroxidase method was used for neurofilament (NF) immunohistochemical staining. Briefly, the $50 \mu \mathrm{m}$ thin frozen sections were treated as follows: 1) incubation in $0.3 \%$ Triton $X-100$ in phosphate-buffered saline (PBS) for $40 \mathrm{~min}$; 2) $0.3 \%$ $\mathrm{H}_{2} \mathrm{O}_{2}$ in PBS for $2 \mathrm{~h}$; 3) 5\% normal donkey serum(NDS) /1\% Bovine serum albumin (BSA) in PBS-T solution (PBS with $0.1 \%$ Tween-20, $\mathrm{pH} 7.0$ ) for $1 \mathrm{~h} ; 4)$ incubation with antineurofilament antibody (1:100; clone 2H3, Developmental Studies Hybridoma Bank) in PBSNDS/BSA (PBS-T with $0.5 \%$ NDS/1\%BSA) for 18-24 h at room temperature (RT; with agitation on a shaker table for all incubation and washing steps); 5) washing with PBSNDS/BSA for $30 \mathrm{~min}$; 6) incubation in 1:2000 anti-mouse IgG (H\&L) secondary antibody Peroxidase conjugate (ROCKLAND) in PBS-NDS/BSA for $2 \mathrm{~h}$ at RT; 7) washing with PBS for $30 \mathrm{~min}$. The sections were then treated with $0.025 \%$ diaminobenzidine (DAB) $/ 0.05 \% \mathrm{H}_{2} \mathrm{O}_{2}$ solution in PBS. The sections were rinsed, mounted on $0.5 \%$ gelatin-coated slides, cover slipped using Pertex and imaged under bright field microscopy.

\subsection{Behavioral testing}

Behavioral testing started when the mice were two months old and the same mice were repeatedly used in the behavioural tests. The testing was carried out in the following order: 1. Open field test, 2. Light-dark box test, 3. Morris water-maze, 4. Elevated plus-maze, 5. Open field+amphetamine. An approximately 10-day washout period was kept between the experiments. 


\subsubsection{Open field test}

The open field test of individual mice was measured in a lit room (ca $2001 \mathrm{x}$ ) for $30 \mathrm{~min}$ in sound-proof photoelectric motility boxes measuring $44.8 \times 44.8 \times 45(\mathrm{H}) \mathrm{cm}$ made of transparent Plexiglas and connected to a computer (TSE, Technical \& Scientific Equipment $\mathrm{GmbH}$, Germany). Before each experiment mice were let to habituate with the experimental room for $1 \mathrm{~h}$. The floor of the boxes was cleaned with 5\% of ethanol and dried thoroughly after each experiment. Computer registered the distance travelled, the number of rearings, and the time spent in the central part of the box.

The effects of d-amphetamine on the locomotor activity were checked using 3-4 months-old $\mathrm{Lsamp}^{-/-} \mathrm{Ntm}^{-/-}$double deficient animals and their wild-type (Wt, $\mathrm{Lsamp}^{+/+} \mathrm{Ntm}^{+/+}$) littermates in the open field test. On Day 1, all mice received an i.p. injection of saline for baseline measurement and on Day 5 all mice received an i.p. injection of $2.5 \mathrm{mg} / \mathrm{kg}$ of d-amphetamine dissolved in saline $30 \mathrm{~min}$ before testing in the motility box. Saline and the d-amphetamine solution were administered at the volume of $10 \mathrm{ml} / \mathrm{kg}$.

\subsubsection{Elevated plus-maze (EPM)}

The elevated plus maze test was carried out as described earlier (Innos et al., 2011) to assay anxiety-related behaviors. In short, the apparatus consisted of two opposite open $(17.5 \times 5 \mathrm{~cm})$ arms without sidewalls and two enclosed arms of the same size with $14 \mathrm{~cm}$ high sidewalls and an end wall. The entire plus maze apparatus was elevated to a height of $30 \mathrm{~cm}$ and placed in a dim room (15 lx in open arms). Testing began by placing the animal on the central platform (5 $\times 5 \mathrm{~cm}$ ) of the maze, facing a closed arm. An arm entry was counted only when all four limbs were within a given arm. Standard 5 min test duration was employed and the sessions were video-recorded. The following parameters were scored by an experienced observer, blind to the experimental group: (1) the number of closed arm entries, (2) the number of open arm entries, (3) the number of protected head dips.

\subsubsection{Light-dark box test}

The light-dark box (TSE, Technical \& Scientific Equipment GmbH, Germany) consisted of a quadratic arena made out of dark Plexiglas on three sides and transparent Plexiglas at the front, measuring $30 \times 30 \times 24(\mathrm{H}) \mathrm{cm}$. Running from front to back of the arena and situated at its midline was a dark Plexiglas wall containing an opening $3.5(\mathrm{~W}) \times 10.0(\mathrm{H}) \mathrm{cm}$, allowing the 
mouse to transfer from one compartment of the arena to the other. The wall divided the arena into a lit chamber (ca 200 lx) and a dark chamber (ca 10 lx, with a lid). The Plexiglas arena was surrounded by a soundproof chamber. The apparatus was located in a quiet, dimly (ca 5 lx) illuminated room. An animal was placed in the dark chamber, facing away from the opening, and released. During a 20 min trial, the latency to enter the lit chamber, time spent in the lit chamber, and the numbers of transitions were measured.

\subsubsection{Morris water maze (MWM) test}

The water maze consisted of a circular pool (diameter $150 \mathrm{~cm})$, escape platform $(16 \mathrm{~cm}$ in diameter), video camera and computer with software (TSE, Technical \& Scientific Equipment $\mathrm{GmbH}$, Germany). The pool (depth $50 \mathrm{~cm})$ was filled with tap water $\left(22^{\circ} \mathrm{C}\right.$, to a depth of 40 $\mathrm{cm})$ that was made opaque by adding a small amount of non-toxic white putty. The escape platform was positioned in the centre of the Southwest quadrant (Q2), $20 \mathrm{~cm}$ from the wall. The water level was $1 \mathrm{~cm}$ above the platform, making it invisible. In each trial, the animals were put into the water, facing the wall, at pseudo-randomly assigned starting positions (East, North, South, or West). The acquisition phase of the experiment consisted of a series of 16 training trials (four trials per day for four consecutive days, inter-trial interval ca $1 \mathrm{~h}$ ). Mice were allowed to search for the platform for a maximum of $60 \mathrm{~s}$ at which time the mice were gently guided to the platform by means of a metal sieve. The mice remained on the platform for ca $15 \mathrm{~s}$. Posters and furniture around the maze served as visual cues. During testing, the room was dimly lit with diffuse white light (20 lx). Distance travelled during the trial, latency to find the submerged platform and swim velocity were registered. We used average values per day, which was obtained by collapsing data of four trials for each animal. On Day 5 the platform was removed for a probe trial. Mice were placed into the water in the Northeast position (Q4) and were allowed to swim for $60 \mathrm{~s}$. Time spent in all four quadrants (Q1, Q2, Q3, Q4) was measured, with time spent in the target quadrant (Q2) where the platform had been located serving as indicator of spatial memory.

\subsection{Data presentation and Statistical Analysis}

The statistical analysis for cell culture experiments was performed using Prism 5 software (Graph Pad, San Diego, CA). Statistical differences for multiple groups were assessed by oneway ANOVA followed by Newman-Keuls post hoc test. Behavioral experiments were analysed with Statistica V12 (Statsoft Inc., Oklahoma, USA). Statistical differences for 
multiple groups were assessed by one-way ANOVA followed by Newman-Keuls post hoc test. The effect of amphetamine in the motility box was analysed with repeated measures ANOVA. Values of $\mathrm{p}<0.05$ were considered significant and experimental values represent the mean \pm SE.

\section{Results}

\subsection{Lsamp and Ntm regulate neuritogenesis in the hippocampal neurons}

We used primary hippocampal cultures derived from Wt, $\mathrm{Lsamp}^{-/}, \mathrm{Ntm}^{-/}$, and $\mathrm{Lsamp}^{-/-} \mathrm{Ntm}^{-/-}$ mice to examine the role of Lsamp and $\mathrm{Ntm}$ in the neuritogenesis processes. At neurite initiation stage, DIV0.25 (6 h post culture) we performed double-immunofluorescent labelling with Phalloidin, the F-actin-binding compound to label actin filaments or "growing protrusions", and neuronal marker, MAP2. While these immunostainings demonstrate that $\mathrm{Lsamp}^{-/}$and Wt hippocampal neurons shared a similar neurite initiation phase (Fig. $\left.1 \mathrm{~A}-\mathrm{H}\right)$, $\mathrm{Ntm}^{-/}$and $\mathrm{Lsamp}^{-/-} \mathrm{Ntm}^{-/}$double-deficient hippocampal neurons had aberrant membrane protrusions and filopodia with one extending neurite, as well as enhanced puncta of F-actin aggregates on the neuron surface (Fig. 1I-P). We performed quantification of the intensity of phalloidin fluorescence on the neuronal surface and found that $\mathrm{Ntm}^{-/-}$hippocampal neurons harboured more actin aggregates compared to all other analysed genotypes $\left(\mathrm{Ntm}^{-/} \mathrm{vs} \mathrm{Wt}\right.$, p $<0.01 ;$ Ntm $^{-/}$vs Lsamp $^{-/}, \mathrm{p}<0.001 ; \mathrm{Ntm}^{-/}$vs Lsamp $^{-/} \mathrm{Ntm}^{-/}$, p<0.05) (Fig 1Q). Our statistical analysis did not reveal any significant changes in phalloidin staining intensity between $\mathrm{Wt}$, $\mathrm{Lsamp}^{-/-}$, and even in $\mathrm{Lsamp}^{-/-} \mathrm{Ntm}^{-/-}$double-deficient hippocampal neurons; for exact values see Supplementary tables S1 and S2).

To view the surface topology of hippocampal neurons at the stage of neurite initiation in detail, we visualised the ultrastructure of neurite precursors at DIV 0.25 using scanning electron microscopy (SEM). SEM images revealed that the cultured hippocampal neurons of different genotypes exhibited distinct features (Fig. 2). At the neurite initiation stage, the cultured neurons attach to the substrate surface and give rise to protrusions to form lamellopodia tipped by filopodia. Wt neurons were spherical smooth cells surrounded by a thin lamellipodial veil and having few protrusions (Fig. 2A, E, I). Some neurons started establishing focal contacts with neighbouring neurons (Fig. 2A-D). The surface of Lsamp $^{-/-}$neurons was also smooth, but 
the extending lamellopodia and filopodia appeared broader and thicker in comparison with those of Wt neurons (Fig. 2E-F, I-J). In agreement with the results of our phalloidin staining, SEM analysis (at DIV 0.25) revealed the excess of aberrant protrusions and lamellopodia in $\mathrm{Ntm}^{-/}$hippocampal neurons. Moreover, the surface was more ruffled compared to Wt and Lsamp $^{-/}$neurons. Outgrowth of a single neurite with excessive branches was also observed in $\mathrm{Ntm}^{-/}$neurons (Fig. 2C, G, K). The surface of $\mathrm{Lsamp}^{-/} \mathrm{Ntm}^{-/}$double deficient hippocampal neurons was even more ruffled and possessed abnormal filopodium with more aggregates at contact points (Fig. 2D, H, L).

In order to investigate how the deficiency of IgLON molecule/s further alters neurite outgrowth and branching, we performed morphometric analysis on primary hippocampal neurons at DIV3.At this stage, the primary hippocampal neurons of all analysed genotypes exhibited welldistinct pyramidal shaped somas extending several neurite processes with multiple levels of branching (Fig. 3A-D). Our morphometric analysis revealed that the number of neurites per neuron was higher in $\mathrm{Ntm}^{-/}$hippocampal neurons compared to Wt neurons $(\mathrm{p}<0.05)$, but that hippocampal neurons from $\mathrm{Lsamp}^{-/}$and $\mathrm{Lsamp}^{-/} \mathrm{Ntm}^{-/}$possessed numbers of neurites per neuron similar to those of the Wt neurons (Fig. 3E). The exact numerical values (mean $\pm \mathrm{SE}$ ) for the morphological phenotyping of neuritogenesis are available in the Supplementary table $\mathrm{S} 1$ and S2. In addition to higher neurite number, the neurons derived from $\mathrm{Ntm}^{-/}$hippocampi also possessed longer neurites compared to $\mathrm{Wt}(\mathrm{p}<0.0001), \operatorname{Lsamp}^{-/-}(\mathrm{p}<0.0001)$, and to the double-deficient $\mathrm{Lsamp}^{-/} \mathrm{Ntm}^{-/-}$neurons ( $\mathrm{p}<0.0001$ ). No statistically significant differences in neurite length could be observed between other genotypes (Fig. 3F).

Quantification of neurite branching points supported our SEM analysis and revealed that the neurites derived from $\mathrm{Ntm}^{-/}$hippocampal neurons branched more than $\mathrm{Wt}(\mathrm{p}<0.01)$ and Lsamp - $(\mathrm{p}<0.01)$ neurons. Also, the number of branching points of $\mathrm{Ntm}^{-/}$neurites was higher compared to $\mathrm{Lsamp}^{-/} \mathrm{Ntm}^{-/-}$neurites ( $\mathrm{p}<0.05$ ), but in the last case the deletion of Lsamp reduced the effect of $\mathrm{Ntm}^{-/}$. Significant difference in the number of branching points was detected between $\mathrm{Lsamp}^{-/} \mathrm{Ntm}^{-/-}$and Wt neurites $(\mathrm{p}<0.05)$, and no significant difference was observed between $\mathrm{Lsamp}^{-/}$and Wt neurons (Fig. 3G). We found no effects on the synaptic number in any of the deficiencies with Lsamp and/or Ntm (Supplementary Fig S3, A-E and Table S4). 


\subsection{Proliferation and apoptosis in Lsamp- and Ntm- deficient hippocampal neurons}

In order to understand the role of Lsamp and Ntm in tissue homeostasis, we compared the rate of proliferation and apoptosis in primary hippocampal cultures at DIV3 derived from Wt, $\mathrm{Lsamp}^{-{ }_{-}}, \mathrm{Ntm}^{-/}$, and $\mathrm{Lsamp}^{-/-} \mathrm{Ntm}^{-/-}$mice. Proliferation rate was assessed by immunolabelling with mitotic phase marker PHH3 (Fig. 4A), immature and developing neurons were visualised by DCX immunostaining, and the nuclei were stained with DAPI. The proliferating cells were calculated as the percentage of proliferative cells (Fig. 4B). The percentage of proliferative cells in $\mathrm{Ntm}^{-/}$cultures was significantly lower compared to those $\operatorname{Lsamp}^{-/-}$cultures $(\mathrm{p}<0.01)$ and $\mathrm{Lsamp}^{-/-} \mathrm{Ntm}^{-/-}(\mathrm{p}<0.001)$ genotypes. The percentage of proliferation in $\mathrm{Lsamp}^{-/} \mathrm{Ntm}^{-/-}$ culture was higher than in Wt $(\mathrm{p}<0.01)$ and in $\mathrm{Ntm}^{-/-}(\mathrm{p}<0.001)$ (Fig.4 B-N; Supplementary table S3).

For the detection of apoptotic cells in hippocampal cultures, we used in-situ TUNEL assay. In Lsamp $^{-/-}$culture, the ratio between all cells to apoptotic cells was significantly higher compared to any other analysed genotype (in all cases p<0.001) (Fig. 5B, E). The percentage of apoptotic cells in $\mathrm{Ntm}^{-/}$, and $\mathrm{Lsamp}^{-/} \mathrm{Ntm}^{-/-}$cultures at DIV3 was similar to Wt culture (Fig. 5A, C-E).

\subsection{Gross anatomy of IgLON/s deficient brains}

Histology of IgLON/s deficient adult brains was visualized by Nissl staining and neurofilament immunostaining. No gross changes in brain anatomy were found by Nissl staining in neither $\mathrm{Lsamp}^{-/}, \mathrm{Ntm}^{-/}$nor $\mathrm{Lsamp}^{-/} \mathrm{Ntm}^{-/}$brains compared to Wt brain sections (Fig. 6A-D). Antineurofilament immunostaining also revealed no obvious disorganisation of the major fiber tracts across genotypes (Fig. 6E-H).

\section{4. Effect of Lsamp and/or Ntm deletions on the mouse behavior}

\subsubsection{Open field test}

Locomotor and exploratory activity was evaluated through the open field activity test using motility boxes. The distance travelled in the motility box during the $30 \mathrm{~min}$ testing was not dependent on genotype $\left(\mathrm{F}_{(3,41)}=2,1, \mathrm{p}=0.12\right)$ (Fig. 7A). However, the number of rearings was 
dependent on genotype $\left(\mathrm{F}_{(3,41)}=3.21, \mathrm{p}<0.05\right)$. Wt and $\mathrm{Ntm}^{-/}$mice displayed approximately twice as much vertical activity as $\mathrm{Lsamp}^{-/}$mice and $\mathrm{Lsamp}^{-/} \mathrm{Ntm}^{-/}$double deficient mice, while, the post hoc comparisons remained at the tendency level (Fig. 7B). Distance in centre $\left(F_{(3,41)}=3.63, p<0.05\right)$ was significantly dependent on genotype. The distance travelled in the centre was significantly longer in $\mathrm{Ntm}^{-/}$mice, who demonstrated anxiolytic-like baseline behaviour in comparison with all the other three groups (Fig. 7C). For the effect of damphetamine on distance travelled in the motility boxes see Supplementary materials (Fig. S4). Shortly, repeated measures ANOVA revealed a significant treatment effect $(F(1,14)=8.1$, $\mathrm{p}=0.013)$, but the genotype effect $(\mathrm{F}(1,14)=1.26, \mathrm{p}=0.28)$ was insignificant and genotype $\mathrm{x}$ treatment effect remained at a tendency level $(\mathrm{F}(1,14)=3.34, \mathrm{p}=0.089)$.

\subsubsection{Light/dark box}

Anxiety-like behaviours in mice were evaluated using light/dark box. The test is based on spontaneous exploratory behaviour in a novel environment which contains a protected area (dark compartment) and a naturally aversive unprotected area (light compartment). None of the three anxiety-related parameters measured in the light/dark test - the number of visits to the light compartment, time spent in the light compartment, and the latency to enter the light compartment - were dependent on genotype (data not shown).

\subsubsection{Elevated plus-maze}

Elevated plus-maze is used to assess anxiety-like behaviour in mice. The test is based on measuring two opposing natural inclinations of rodents. On one hand, they tend to avoid unprotected open arms of the maze, but on the other hand, they are inclined to explore novel environments. Thus, the more activity in open arms, the less anxious the mouse is construed to be. In the current study wild-type mice showed no activity in the open arms of the testing apparatus, which is in line with our previous experiments, where we have observed wild-type mice performing few or no open arm entries (Innos et al, 2011; Innos et al, 2012). This behaviour can be considered normally adaptive in mice, as mice taken from their home cages will generally show a pattern of behaviour characterized by open-arm avoidance and consistent preference for the closed arms (Bourin et al, 2007). The number of entries mice 
made into the central platform of the EPM was not dependent on genotype $\left(F_{(3,38)}=2,4275\right.$, $\mathrm{p}=0.08$ ). The time (s) that mice spent in the central platform of the EPM was also not dependent on genotype $\left(\mathrm{F}_{(3,38)}=1,4272, \mathrm{p}=0.25\right)$. On average, wild-type mice spent $15 \pm 4.4$ seconds, $\mathrm{Lsamp}^{-/-}$mice $12.7 \pm 2.7$ seconds, $\mathrm{Ntm}^{-/}$mice $21.2 \pm 3.6$ seconds and $\mathrm{Lsamp}^{-/-} \mathrm{Ntm}^{-/-}$ mice $22 \pm 5$ seconds in the central platform of the EPM. The number of closed arm entries was dependent of genotype $\left(\mathrm{F}_{(3,38)}=4.05, \mathrm{p}=0.014\right)$ and according to post hoc analysis Wt mice performed significantly less closed arm entries than $\mathrm{Lsamp}^{-/} \mathrm{Ntm}^{-/-}$mice ( $\left.\mathrm{p}=0.016\right)$ (Fig. 7D). The number of open arm entries was also dependent on genotype $\left(F_{(3,38)}=4.79, p=0.006\right)$ and the post hoc analysis showed that $\mathrm{Lsamp}^{-/-} \mathrm{Ntm}^{-/-}$mice performed significantly more open arm entries than Wt $(\mathrm{p}=0.008)$ and $\mathrm{Ntm}^{-/}$mice $(\mathrm{p}=0.026)$ and the difference compared to Lsamp ${ }^{-/}$ mice verged on significance $(\mathrm{p}=0.06)$ (Fig. 7E ). Protected head dips were dependent on genotype $\left(\mathrm{F}_{(3,38)}=2.9, \mathrm{p}=0.047\right)$, but no post hoc differences were detected (Fig. 7F).

\subsubsection{Morris water maze}

We used the Morris water maze to study spatial learning and memory in mice (Jeffery and Morris, 1993). The mice have to rely on visual cues placed around the perimeter of the swimming arena to find the location of the submerged escape platform. Spatial learning is evaluated against repeated trials and the reference memory is analysed by preference for the platform area when the platform is absent.

For the latency to find the submerged platform in Days 1-4, there was a clear learning (day) effect $\left(\mathrm{F}_{(3,114)}=62.6, \mathrm{p}<0.0001\right)$ and both genotype effect $\left(\mathrm{F}_{(3,38)}=2.67, \mathrm{p}=0.061\right)$ and genotype $\mathrm{x}$ day interaction $\left(\mathrm{F}_{(9,114)}=1.91, \mathrm{p}=0.058\right)$ approached significance with $\mathrm{Lsamp}^{-/} \mathrm{Ntm}^{-/}$double deficient mice tending to display longer latencies in Days 2 and 4 (Fig. 7G-H). However, this should not be interpreted as learning deficiency as there were clear differences in swimming speed between the genotype groups (see below). Therefore, distance to the platform is a more objective parameter for expressing the learning curve. The distance was likewise very significantly dependent on day $\left(\mathrm{F}_{(3,114)}=46.19, \mathrm{p}<0.0001\right)$, but not genotype $\left(\mathrm{F}_{(3,38)}=1.67\right.$, $\mathrm{p}=0.19)$. Genotype $\mathrm{x}$ day interaction was significant $\left(\mathrm{F}_{(9,114)}=2.14, \mathrm{p}=0.031\right)$, but post hoc comparisons revealed differences between the groups only in the 1st day (most notably, Wt vs $\mathrm{Lsamp}^{-/-} \mathrm{Ntm}^{-/}$mice $\left.\mathrm{p}=0.004\right)$, which basically reflects differences in swimming speed. Probe trial on Day 5 confirmed the lack of learning deficiency in deficient mice groups as all the 
genotypes clearly preferred the target quadrant (Fig. 7I) and there were no differences between the groups $\left(\mathrm{F}_{(3,38)}=0.87, \mathrm{p}=0.47\right)$. Swimming speed was very significantly dependent on genotype $\left(\mathrm{F}_{(3,38)}=24.19, \mathrm{p}<0.0001\right)$; both single deficient groups swam slower than wild-type mice and the simultaneous deletion of Lsamp and Ntm genes further aggravated swimming speed deficiency compared to single deficient mice (Fig. 7J). 


\section{Discussion}

This study investigated the effects of two neural adhesion molecules at different functional levels of the nervous system, such as neuronal development and behaviour. Combining mutations within the IgLON family gave us a spectrum of phenotypes, which enabled us to show differential interactions between the two members of IgLONs for the performance of behaviour along with underlying neuronal development. Our results are the first to demonstrate that members of the IgLON family of adhesion molecules can regulate the initiation of neurite sprouting at early stages, even cell-autonomously, without existing adhesion between the cells.

Our initial observation was the surprising discovery that Ntm/Lsamp double-knockout mice were not distinguishable from their wild-type, $\mathrm{Lsamp}^{-\mathrm{I}_{-}}$or $\mathrm{Ntm}^{-/-}$littermates. We found no gross vision or hearing deficiencies nor significant changes in body-weight in double mutant mice. The behavioural profiling indicated an interplay between Lsamp and Ntm, which varied across specific tests. The phenotypes characteristic of the single mutant lines, such as reduced swimming speed in Morris water maze and anxiolytic phenotype in the elevated plus maze, were magnified in $\mathrm{Lsamp}^{-/} \mathrm{Ntm}^{-/-}$mice, indicating that in certain traits the deletion of both Lsamp and Ntm induces a more prominent phenotype than single gene deficiency. However, the behavioural phenotypes apparent in single mutant lines could also stay the same or disappear in double-mutant mice.

\subsection{Lsamp and Ntm regulate neuritogenesis in the hippocampal neurons}

In order to study morphological changes in neural circuitry underlying behavioural deviations, the in vitro studies using neurons derived from the hippocampi of $\mathrm{Lsamp}^{-/}, \mathrm{Ntm}^{-/} \mathrm{or} \mathrm{Lsamp}^{-/}$ $\mathrm{Ntm}^{-/}$mice was performed in parallel with behavioural studies. The coexpression of Lsamp and Ntm in the same neurons has been shown in cultured hippocampal neurons (Gil et al., 2002). Additionally, both Lsamp and Ntm are located at the surfaces of neuronal somata, dendrites and axons during the first post-natal weeks (Zacco et al., 1990; Chen et al., 2001). The earliest known phase expression of Lsamp and Ntm in the hippocampal region has been detected prenatally at around E16-E18 (Pimenta et al., 1995; Struyk et al., 1995; Diez-Roux et al., 2011). Here we investigated the development of new-born hippocampal cells isolated from P0-1 pups at stage 1 ( $6 \mathrm{~h}$ after plating) and at stage 3 ( 3 days after plating). The expression of Lsamp and Ntm in the cultured hippocampal primary neurons at DIV3 and in P3 mouse 
hippocampus was verified to support the reliability of our in vitro model (Supplementary fig. S5).

During neurite initiation stage $1, \mathrm{Ntm}^{-/}$hippocampal neurons exhibited significantly increased neurite sprouting indicated by more F-actin rich areas compared with any other genotype (Wt,

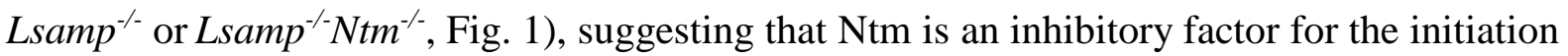
of neuritogenesis. Deletion of Lsamp alone had no effect on the initial dynamics of the cytoskeleton; however, the robust effect of deleting Ntm alone disappeared if Lsamp was deleted as well ( $\mathrm{Lsamp}^{-/} \mathrm{Ntm}^{-/}$double-deficient neurons). Accordingly, we hypothesize that Lsamp is an enhancer of processes that are correlated with actin accumulation in the initiation of filopodium, as premature neuritogenesis in $\mathrm{Ntm}^{-/}$neurons is exhibited only in the presence of Lsamp. Illustrative SEM analysis enabled us to see 3D images confirming longer and more branched initial neurite protrutions of $\mathrm{Ntm}^{-/}$hippocampal neurons and alterations in the cell

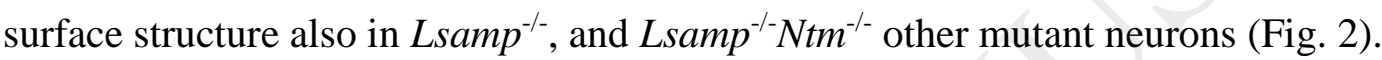

Morphometric analyses revealed that premature initiation of neurite sprouting in $\mathrm{Ntm}^{-/}$ hippocampal neurons is consequently seen in the accelerated elongation of neurites, which are more branched. Moreover, the number of neurites was also increased in Ntm-deficient neurons in comparison with Wt neurons (Fig. 3). Again, Lsamp ${ }^{-/}$neurons did not show significant changes in neurite outgrowth and branching compared to Wt neurons. However, the deletion of Lsamp along with $\mathrm{Ntm}$ diminished the effect of $\mathrm{Ntm}^{-/}$genotype and the morphology of $\mathrm{Lsamp}^{-1-} \mathrm{Ntm}^{-/-}$neurons was not different from Wt neurons. We therefore propose that $\mathrm{Ntm}$ acts as a negative factor for neurite outgrowth and branching. The disappearance of the effect of $\mathrm{Ntm}$ deletion in $\mathrm{Lsamp}^{-\mathrm{N}_{-}} \mathrm{Ntm}^{-/-}$neurons suggests that Lsamp might be required as an enhancing factor for neuritogenesis in $\mathrm{Ntm}^{-/-}$neurons. In the current study we focused on the cellular morphology reflecting alterations in neuritogenesis and the differential quantification of axons and dendrites was not our focus in any phases of the study as initially, the sprouting neurites lack the defining features of an axon or dendrite (Silva and Dotti, 2002). However, while estimating the general appearance of the cultures, we observed that cellular polarization is clearly noticeable in $\mathrm{Ntm}^{-/}$neurons at DIV3: the outgrowth of one major neurite is faster in comparison with Wt neurons, indicating the acceleration of neuronal maturation. On the contrary, it was not possible to distinguish one major neurite in any of the double-mutant Lsamp $^{-/-} \mathrm{Ntm}^{-/-}$neurons. 


\subsection{The interplay of Lsamp and Ntm in tissue homeostasis}

Coordination between cellular proliferation and apoptosis is necessary for maintaining the delicate balance of internal development, tissue homeostasis, and growth to avoid uncontrolled cellular growth as seen in cancers. Lsamp has been proposed to function as a tumoursuppressor, since it has been found to be downregulated in several cancerous tissues such as acute myeloid leukaemia (Coccaro et al., 2015), osteosarcoma (Barøy et al., 2014) and clear cell renal carcinomas (Chen et al., 2003). The role of Ntm in tumorigenesis is not straightforward; it has often been shown to have opposite impact compared with Lsamp and other IgLONs. Namely, Lsamp has been shown to be downregulated in ovarian cancer (Ntougkos et al., 2013) and in invasive prostate tumours (Pascal et al., 2009). The expression of Ntm, on the contrary, has been shown to be significantly increased in the same samples.

In order to reveal the potential interactions of Lsamp and Ntm in tissue homeostasis, we examined cellular proliferation and apoptosis in $\mathrm{Lsamp}^{-/}, \mathrm{Ntm}^{-/-}$and $\mathrm{Lsamp}^{-/-} \mathrm{Ntm}^{-/}$doubledeficient hippocampal cultures. Previously it has been shown that overexpression of Lsamp in cancerous cell lines reduces the rate of proliferation (Barøy et al., 2014); here we showed that the deletion of Lsamp alone did not affect the proliferation rate. However, our double deficient model enabled us to track the influence of Lsamp in combination with Ntm (Fig. 4). Ntm ${ }^{-/}$ hippocampal culture exhibits less proliferation compared to the Wt neurons, at the same time, additional deletion of Lsamp ( $\mathrm{Ssamp}^{-/-} \mathrm{Ntm}^{-/}$culture) results in disappearance of the previous effect indicating that $\mathrm{Ntm}$ acts as a positive factor inducing proliferation but only in the presence of Lsamp. Interestingly, $\mathrm{Lsamp}^{-/} \mathrm{Ntm}^{-/-}$results in enhancement of the proliferation rate, indicating that the direction of the impact of $\mathrm{Ntm}$ is dependent on Lsamp.

Earlier reports have shown that overexpression of Lsamp does not affect apoptosis in cancerous cell lines (Chen et al., 2003; Barøy et al., 2014). Importantly, we detected a significant decrease of apoptotic cells in Lsamp-deficient neuronal cultures, whereas Ntm deficiency and Lsamp/Ntm double-deficiency had no effect on apoptotic cell percentage. We could conclude that Lsamp induces apoptosis only in the presence of Ntm which thus seems to be an important interaction partner for Lsamp. Our result that Lsamp can act as a positive factor in the apoptosis-inducing cellular pathway can be one explanation for the tumour-suppressor role, often reported for Lsamp. The impact of Ntm for cellular growth seems to be dependent on the binding partners or specific context. Here we showed that the direction of the impact of Ntm is dependent on Lsamp. Indeed, whereas in most experiments $\mathrm{Ntm}$ has been found to be 
upregulated in cancerous tissues (Ntougkos et al., 2005; Makowski et al., 2014; Ogawa et al., 2006). Other data exist that Ntm can also be downregulated together with Lsamp in some tumours, such as medulloblastoma and bladder cancer (Barøy et al., 2014). Taken together, Lsamp and Ntm work together in balancing cellular homeostasis indicating their combined effects during development and in malignant cellular processes.

\subsection{Single and combined effects of $\mathrm{Ntm} / \mathrm{Lsamp}$-deficiency on the behaviour}

We found no evidence of gross changes in brain anatomy in $\mathrm{Lsamp}^{-/}, \mathrm{Ntm}^{-/} \mathrm{or} \mathrm{Lsamp}^{-/} \mathrm{Ntm}^{-/-}$ mice. The general organization of the cortex, hippocampus and other subcortical areas did not reveal gross changes (Fig. 6). However, behavioural testing of $\mathrm{Lsamp}^{-{ }_{-}}, \mathrm{Ntm}^{- \text {}_{-}}$, and $\mathrm{Lsamp}^{-/-}$ $\mathrm{Ntm}^{-/-}$double-deficient mutant mice exposed phenotypes indicating combined operating of Lsamp and Ntm.

The current study revealed no significant differences in general locomotor activity between the four groups in the open field test; although $\mathrm{Ntm}^{-/}$mice spent more time and travelled a longer distance in the central area of the open field test. In our previous study (Mazitov et al., 2017) the same effect in $\mathrm{Ntm}^{-/}$mice was observed as a tendency that did not meet statistical significance. Deletion of Lsamp alone had no effect on the exploratory activity in the centre of the open field, consistent with our previous results (Innos et al, 2011); furthermore, the significant exploratory effect of deleting Ntm alone disappeared if Lsamp was deleted as well (in $\mathrm{Lsamp}^{-/-} \mathrm{Ntm}^{-/}$double-deficient mice, (Fig. 7C)). Again, we can hypothesize that Lsamp is an enhancer of the exploratory activity in the central area of the open field as increased time in the centre in $\mathrm{Ntm}^{-1}$ mice is apparent only in the presence of Lsamp. Rearings in the open field, reflecting vertical exploratory activity, were suppressed in Lsamp $^{-/}$mice and at normal level in $\mathrm{Ntm}^{-/}$mice, confirming our previous studies (Innos et al., 2011; Mazitov et al., 2017). Thus, the decreased frequency of rearings seen in $\mathrm{Lsamp}^{-/-} \mathrm{Ntm}^{-/}$double deficient mice was apparently the effect of Lsamp deficiency (Fig. 7B). We have previously demonstrated decreased sensitivity to the locomotor stimulating effects of amphetamine in both $\mathrm{Lsamp}^{-/}$and $\mathrm{Ntm}^{-/-}$ animals (Innos et al., 2013; Mazitov et al., 2017), whereas the magnitude of change was much larger in $\mathrm{Lsamp}^{-/-}$animals. This difference in amphetamine sensitivity was most prominent in young (2-3 months old) unhandled animals and tended to decrease in older and/or handled animals. Our present results also show a tendency towards decreased sensitivity to 
amphetamine in $\mathrm{Lsamp}^{-/-} \mathrm{Ntm}^{-/}$double deficient mice. However, because of the technical difficulties related to obtaining a sufficient number of double-mutant mice we could not use young unhandled mice, and the experiment was performed in handled 4 months old animals. Keeping this shortcoming in mind, we can conclude that further deletion of the Ntm gene does not induce drastic changes in sensitivity to amphetamine as compared to Lsamp ${ }^{-/}$animals (see Supplementary Fig. S7 for more detailed information).

Elevated plus-maze was used to detect genotype-dependent changes in anxiety. Here we show that $\mathrm{Lsamp}^{-/-} \mathrm{Ntm}^{-/}$double-deficient mice displayed robust reduction in anxiety-like behaviour in the EPM compared to all the other genotypes. Lsamp ${ }^{-/-} \mathrm{Ntm}^{-/}$double deficient mice performed more open arm entries and spent longer time in open arms than wild-type mice and single mutants (Fig. 7E). We propose that that the prominent anxiolytic phenotype in Lsamp ${ }^{-/}$ $\mathrm{Ntm}^{-/}$double deficient mice, which may reflect the magnification of reduced anxiety traits in single mutants, can be seen as a model of the architecture of a polygenic psychiatric disease: the effects of single genes behind maladaptive behavioral traits are magnified in the disturbance of multiple loci until the behavioural deviation meets diagnostic criteria. It has repeatedly been demonstrated that $\mathrm{Lsamp}^{-/}$mice are more active and tend to spend more time on the open arm of the EPM (Catania et al., 2008; Innos et al., 2011, 2012). While we have not seen significant behavioural changes in the EPM in $\mathrm{Ntm}^{-/}$mice, $\mathrm{Ntm}^{-/}$mice tend to spend more time in the centre of the open field, representing another indication of reduced anxiety as the centre is normally avoided by wild-type mice (Bourin et al, 2007). It is not always clear in the behavioural tests whether mice display reduced anxiety, exaggerated behavioral activation or increased locomotor activity (Catania et al, 2008, Innos et al., 2011). Taken together, these traits (for example, increased time in the open arm of the EPM and in the centre of the open field) reflect the multidimensional structure of anxiety-related behaviour in mouse (Carola et al, 2002).

In order to measure hippocampal-dependent learning and memory, we tested mice in the Morris water maze. In agreement with our previous studies done on $\mathrm{Lsamp}^{-/}$and $\mathrm{Ntm}^{-/}$mice (Innos et al., 2011; Mazitov et al., 2017), we found no gross learning impairment neither in single gene deficiency nor in $\mathrm{Lsamp}^{-/} \mathrm{Ntm}^{-/}$double deficient mice (Fig. 7G-J). Interestingly, we saw a magnified deficiency effect in swimming speed: reduced swimming speed phenotype in both Lsamp and Ntm deficient mice which is further enhanced in double deficient mice (Fig. 7J). The reduced swimming speed phenotype is very persistent in Lsamp- 
/- mice, despite normal performance in fine motor tests such as rota rod or beam walking test (Innos et al., 2011). Also, we could not detect any obvious motor deficits involving muscle strength and coordination in $\mathrm{Lsamp}^{-/-} \mathrm{Ntm}^{-/}$mice (Supplement S8). The role of IgLONs in the cellular connections behind the regulation of swimming movements needs to be clarified in future studies.

\section{Conclusions}

Correct connections between neurons are essential for normal brain development and appropriate synapse formation. This morphological and physiological connectivity in the brain is thought to underlie appropriate neural conductivity and normal behaviours. Abnormal integrity of neural tracts, leading to disrupted functional brain connectivity, has been suggested to be an endophenotype for several psychiatric disorders, such as schizophrenia (Li et al., 2017). Certain cell membrane moieties, such as the IgLON proteins, are critical for such interactions between neural cells. We propose that the complementary involvement of both Lsamp and Ntm molecules are critical not only for intracellular adhesion, but also for neurite differentiation, wherein they balance the speed and rate of neurite sprouting, as well as neurite elongation and branching. Based on current data, it is complicated to draw clear connections between the neuronal morphology and behavioural profile. However, the findings that the deletional effect of one $\operatorname{IgLON}$ protein $(\mathrm{Ntm})$ is dependent on the presence of another family member (Lsamp) can be seen both at the neuronal level (premature neuritogenesis) and at the behavioural level (increased activity in the centre of the open field). Future studies are needed to confirm if there is a direct causal relationship between alterations in the development seen in our mutant cell models and maladaptive risky behaviours in later life. However, our data provides the evidence that Lsamp and Ntm neural adhesion molecules act synergistically, each forming the context for the work of the other in the regulation of neural circuit formation which manifests both at the level of neuronal morphology and behaviour.

\section{Funding}

This study was supported by an institutional investigation grant from the Estonian Research Council IUT20-41 (E. Vasar) and personal investigation grant from the Estonian Research 
Council PUT129 (M.-A. Philips). This research was also supported by the European Union through the European Regional Development Fund (Project No. 2014-2020.4.01.150012) and from the European Union's Horizon 2020 research and innovation programme under grant agreement 692202. 


\section{References}

Akeel, M., McNamee, C. J., Youssef, S., Moss, D., 2011. DIgLONs inhibit initiation of neurite outgrowth from forebrain neurons via an IgLON-containing receptor complex. Brain Res 1374, 27-35. doi: 10.1016/j.brainres.2010.12.028.

Argyelan, M., Ikuta, T., DeRosse, P., Braga, R.J., Burdick, K.E., John, M., Kingsley, P.B., Malhotra, A.K., Szeszko, P.R., 2014. Resting-State fMRI connectivity impairment in schizophrenia and bipolar disorder. Schizophr Bull 40, 100-10. doi: 10.1093/schbul/sbt092.

Baj, G., Patrizio, A., Montalbano, A., Sciancalepore, M., Tongiorgi, E., 2014. Developmental and maintenance defects in Rett syndrome neurons identified by a new mouse staging system in vitro. Front Cell Neurosci 8, 18. doi: 10.3389/fncel.2014.00018.

Bakos, J., Bacova, Z., Grant, S. G., Castejon, A.M., Ostatnikova, D., 2015. Are Molecules Involved in Neuritogenesis and Axon Guidance Related to Autism Pathogenesis? Neuromol Med 17, 297-304. doi: 10.1007/s12017-015-8357-7.

Barøy, T., Kresse, S. H., Skårn, M., Stabell, M., Castro, R., Lauvrak, S., Llombart-Bosch, A., Myklebost, O., Meza-Zepeda, L. A., 2014. Reexpression of LSAMP inhibits tumor growth in a preclinical osteosarcoma model. Mol Cancer 13, 93. doi: 10.1186/1476-4598-1393.

Behan, A. T., Byrne, C., Dunn, M. J., Cagney, G., Cotter, D. R., 2009. Proteomic analysis of membrane micro domain-associated proteins in the dorsolateral prefrontal cortex in schizophrenia and bipolar disorder reveals alterations in LAMP, STXBP1 and BASP1 protein expression. Mol. Psychiatry 14, 601-613.doi:10.1038/mp.2008.7

Bourin, M., Petit-Demoulière, B., Dhonnchadha, B.N., Hascöet, M., 2007. Animal models of anxiety in mice. Fundam Clin Pharmacol. 21(6), 567-574. doi: 10.1111/j.14728206.2007.00526.x

Coccaro, N., Zagaria, A. , Tota, G. , Anelli, L. , Orsini, P. , Casieri, P. , Cellamare, A., Minervini, A., Impera, L., Minervini, C. F., et al., 2015. Overexpression of the LSAMP and TUSC7 genes in acute myeloid leukemia following microdeletion/duplication of chromosome 3. Cancer Genet. 10, 517-522. 10.1016/j.cancergen.2015.07.006

Carola, V., D'Olimpio, F., Brunamonti, E., Mangia, F., Renzi, P., 2002. Evaluation of the elevated plus-maze and open-field tests for the assessment of anxiety-related behaviour in inbred mice. Behav Brain Res. 134(1-2), 49-57. doi: 10.1016/S0166-4328(01)00452-1

Catania, E. H., Pimenta, A., Levitt, P., 2008. Genetic deletion of Lsamp causes exaggerated behavioral activation in novel environments. Behav. Brain Res. 188, 380-390.

Chatterjee, S., Sikdar, S.K., 2014. Corticosterone targets distinct steps of synaptic transmission via concentration specific activation of mineralocorticoid and glucocorticoid receptors. J Neurochem 128(4), 476-490. doi: 10.1111/jnc.12478.

Chen, S., Gil, O., Ren, Y.Q., Zanazzi, G., Salzer, L.J., Hillman, E.D., 2001. Ntm expression during cerebellar development suggests roles in axon fasciculation and synaptogenesis. $\mathrm{J}$ 
Neurocytol 30, 927-937. doi: 10.1023/A:1020673318536.

Chen, J., Lui, W. O., Vos, M.D., Clark, G.J., Takahashi, M., Schoumans, J., Khoo, S.K., Petillo, D., Lavery, T., Sugimura, J., et al., 2003. The t(1;3) breakpoint-spanning genes LSAMP and NORE1 are involved in clear cell renal cell carcinomas. Cancer Cell 4(5), 405413. doi: http://dx.doi.org/10.1016/S1535-6108(03)00269-1.

Craig, A. M., Banker, G., 1994. Neuronal polarity. Annu Rev Neurosci 17, 267-310. doi: 10.1146/annurev.ne.17.030194.001411.

da Silva, J.S., Dotti, C.G., 2002. Breaking the neuronal sphere: regulation of the actin cytoskeleton in neuritogenesis. Nat Rev Neurosci 3, 694-704. doi: 10.1038/nrn918.

Diez-Roux, G., Banfi, S., Sultan, M., Geffers, L., Anand, S., Rozado, D., Magen, A., Canidio, E., Pagani, M., Peluso, I., et al., 2011. A high-resolution anatomical atlas of the transcriptome in the mouse embryo. PLoS Biol 9 (1), e1000582. doi: 10.1371/journal.pbio.1000582.

Flynn, K.C., 2013. The cytoskeleton and neurite initiation. Bioarchitecture 3(4), 86-109. doi: 10.4161/bioa.26259.

Gil, O.D., Zhang, L., Chen, S., Ren, Y.Q., Pimenta, A., Zanazzi, G., Hillman, D., Levitt, P., Salzer, J.L., 2002. Complementary Expression and Heterophilic Interactions between IgLON Family Members Ntm and LAMP. J Neurobiol 51, 190-204. doi: 10.1002/neu.10050.

Hashimoto, T., Maekawa, S., Miyata, S., 2009. IgLON cell adhesion molecules regulate synaptogenesis in hippocampal neurons. Cell Biochem Funct 27, 496-498. doi: $10.1002 / \mathrm{cbf} .1600$.

Heinla, I., Leidmaa, E., Kongi, K., Pennert, A., Innos, J., Nurk, K., Tekko, T., Singh, K., Vanaveski, T., Reimets, R., et al., 2015. Gene expression patterns and environmental enrichment-induced effects in the hippocampi of mice suggest importance of Lsamp in plasticity. Front Neurosci 9, 205. doi: 10.3389/fnins.2015.00205.

Innos, J., Philips, M.A., Leidmaa, E., Heinla, I., Raud, S., Reemann, P., Plaas, M., Nurk, K., Kurrikoff, K., Matto, V., et al., 2011. Lower anxiety and a decrease in agonistic behaviour in Lsamp-deficient mice. Behav Brain Res 217, 21-31. doi:

10.1016/j.bbr.2010.09.019.

Innos, J., Philips, M.A., Raud, S., Lilleväli, K., Kõks, S., Vasar, E., 2012. Deletion of the Lsamp gene lowers sensitivity to stressful environmental manipulations in mice. Behav Brain Res 228, 74-81. doi: 10.1016/j.bbr.2011.11.033.

Innos, J., Koido, K., Philips, M.A., Vasar, E., 2013. Limbic system associated membrane protein as a potential target for neuropsychiatric disorders. Front Pharmacol 4, 32. doi: 10.3389/fphar.2013.00032.

Innos, J., Leidmaa, E., Philips, M.A., Sütt, S., Alttoa, A., Harro, J., Kõks S, Vasar E., 2013. Lsamp (-/-) mice display lower sensitivity to amphetamine and have elevated 5-HT turnover. Biochem Biophys Res Commun 430, 413-418. doi: 10.1016/j.bbrc.2012.11.077. 
Jeffery, K.J., Morris, R.G.M., 1993. Cumulative long-term potentiation in the rat dentate gyrus correlates with, but does not modify, performance in the water maze. Hippocampus 3 , 133-140. doi: 10.1002/hipo.450030205.

Karis, K., Eskla, K. L., Kaare, M., Täht, K., Tuusov, J., Visnapuu, T., Innos, J., Jayaram, M.,Timmusk, T., Weickert, C. S., Väli, M., Vasar, E., Philips, M.A., 2018. Altered expression profile of $\operatorname{IgLON}$ family of neural cell adhesion molecules in the dorsolateral prefrontal cortex of schizophrenic patients. Front. Mol. Neurosci. doi: 10.3389/fnmol.2018.00008.

Keller, F., Rimvall, K., Barbe, M. F., Levitt, P., 1989. A membrane glycoprotein associated with the limbic system mediates the formation of the septo-hippocampal pathway in vitro. Neuron 3, 551-561.

Kim, H., Hwang, J.S., Lee, B., Hong, J., Lee, S., 2014. Newly Identified Cancer-Associated Role of Human Neuronal Growth Regulator 19 (NEGR1). J Cancer 5(7), 598-608. doi: 10.7150/jca.8052.

Koido, K., Traks, T., Balotsev, R., Eller, T., Must, A., Koks, S., Maron, E., Toru, I., Shlik, J., Vasar, V., Vasar, E., 2012. Associations between LSAMP gene polymorphisms and major depressive disorder and panic disorder. Translational psychiatry 2, e152. doi: $10.1038 /$ tp.2012.74.

Koido, K., Janno, S., Traks, T., Parksepp, M., Ljubajev, Ü., Veiksaar, P., Must, A., Shlik, J., Vasar, V., Vasar, E., 2014. Associations between polymorphisms of LSAMP gene and schizophrenia. Psychiatry Res 215, 797-798. doi: 10.1016/j.psychres.2014.01.016.

Kolodkin, A. L., Tessier-Lavigne, M., 2011. Mechanisms and molecules of neuronal wiring: a primer. Cold Spring Harb Perspect Biol 3 (6). doi: 10.1101/cshperspect.a001727.

Lang, B., Pu, J., Hunter, I., Liu, M., Martin-Granados, C., Reilly, T.J., Gao, G. D., Guan, Z. L., Li, W. D., Shi, Y.Y., et al., 2014. Recurrent deletions of ULK4 in schizophrenia: a gene crucial for neuritogenesis and neuronal motility. J Cell Sci 127, 630-640. doi: $10.1242 /$ jcs. 137604 .

Li, P., Fan, T.T., Zhao, R.J., Han, Y., Shi, L., Sun, H.Q., Chen, S.J., Shi, J., Lin, X., Lu, L., 2017. Altered Brain Network Connectivity as a Potential Endophenotype of Schizophrenia Sci Rep. 7, 5483. doi: 10.1038/s41598-017-05774-3.

Liu, F., Arias-Vasquez, A., Sleegers, K., Aulchenko, Y.S., Kayser, M. , Sanchez-Juan, P., Feng, B.J., Bertoli-Avella, A.M., van Swieten, J., Axenovich, T. I., et al., 2007. A genomewide screen for late-onset Alzheimer disease in a genetically isolated Dutch population. Am. J. Hum. Genet. 81 (1), 17-31.

Makowski, L., Zhou, C., Zhong, Y., Kuan, P. F., Fan, C., Sampey, B.P., Difurio, M., BaeJump, V. L., 2014. Obesity increases tumor aggressiveness in a genetically engineered mouse model of serous ovarian cancer. Gynecol Oncol 133(1), 90-97. doi:

10.1016/j.ygyno.2013.12.026. 
Mann, F., Zhukareva, V., Pimenta, A., Levitt, P., Bolz, J., 1998. Membrane-associated molecules guide limbic and non limbic thalamocortical projections. J Neurosci 18, 94099419.

Mazitov,T., Bregin, A., Philips, M. A., Innos, J., Vasar, E., 2017. Deficit in emotional learning in neurotrimin knockout mice. Behav Brain Res 28, 311-318. doi: 10.1016/j.bbr.2016.09.064.

Minhas, H. M., Pescosolido, M. F., Schwede, M., Piasecka, J., Gaitanis, J., Tantravahi, U., Morrow, E. M., 2013. An unbalanced translocation involving loss of 10q26.2 and gain of $11 \mathrm{q} 25$ in a pedigree with autism spectrum disorder and cerebellar juvenile pilocytic astrocytoma. Am J Med Genet A 161, 787-791. doi: 10.1002/ajmg.a.35841.

Miyata, S., Matsumoto, N., Taguchi, K., Akagi, A., Iino, T., Funatsu, N., Maekawa, S., 2003. Biochemical and ultrastructural analyses of IgLON cell adhesion molecules, Kilon and OBCAM in the rat brain. Neuroscience 117, 645-658. http://dx.doi.org/10.1016/S03064522(02)00873-4.

Must, A., Tasa, G., Lang, A., Vasar, E., Kõks, S., Maron, E., Väli, M., 2008. Association of limbic system-associated membrane protein (LSAMP) to male completed suicide. BMC Med Genetics 9, 34. doi: 10.1186/1471-2350-9-34.

Ntougkos, E., Rush, R., Scott, D., Frankenberg, T., Gabra, H., Smyth, J. F., Sellar, G. C., 2005. The IgLON family in epithelial ovarian cancer: expression profiles and clinicopathologic correlates. Clin Cancer Res 11, 5764-5768. doi: 10.1158/1078-0432.CCR04-2388.

Ogawa, K., Utsunomiya, T., Mimori, K., Tanaka, F., Haraguchi, N., Inoue, H., Murayama, S., Mori, M., 2006. Differential gene expression profiles of radioresistant pancreatic cancer cell lines established by fractionated irradiation. Int J Oncol 28(3), 705-713. doi: 10.3892/ijo.28.3.705.

Pan, Y., Wang, K. S., Aragam, N., 2010. NTM and NR3C2 polymorphisms influencing intelligence: family-based association studies. Prog Neuropsychopharmacol Biol Psychiatry 35(1), 154-160. doi: 10.1016/j.pnpbp.2010.10.016.

Pascal, L.E., Vêncio, R.Z., Page, L.S., Liebeskind, E.S., Shadle, C.P., Troisch, P., Marzolf, B., True, L.D., Hood, L.E., Liu, A.Y., 2009. Gene expression relationship between prostate cancer cells of Gleason 3, 4 and normal epithelial cells as revealed by cell type-specific transcriptomes. BMC Cancer 9, 452. doi: 10.1186/1471-2407-9-452.

Philips, M.A., Lilleväli, K., Heinla, I., Luuk, H., Hundahl, C.A., Kongi, K., Vanaveski, T., Tekko, T., Innos, J., Vasar, E., 2015. Lsamp is implicated in the regulation of emotional and social behavior by use of alternative promoters in the brain. Brain Struct Funct 220, 1381-1393. doi: 10.1007/s00429-014-0732-x.

Pimenta, A.F., Zhukareva, V., Barbe, M.F., Reinoso, B. S., Grimley, C., Henzel, W., Fischer, I., Levitt, P., 1995. The limbic system-associated membrane-protein is an Ig superfamily member that mediates selective neuronal growth and axon targeting. Neuron 15, 287-297. doi: 10.1016/0896-6273(95)90034-9. 
Pischedda, F., Szczurkowska, J., Cirnaru, M. D., Giesert, F., Vezzoli, E., Ueffing, M., Sala, C., Francolini, M., Hauck, S.M., Cancedda, L., Piccoli, G., 2014. A cell surface biotinylation assay to reveal membrane-associated neuronal cues: negr1 regulates dendritic arborization. Mol Cell Proteomics 13, 733-748. doi: 10.1074/mcp.M113.031716.

Qiu, S., Champagne, D. L., Peters, M., Catania, E. H., Weeber, E. J., Levitt, P., Pimenta, A.F., 2010. Loss of limbic system-associated membrane protein leads to reduced hippocampal mineralocorticoid receptor expression, impaired synaptic plasticity, and spatial memory deficit. Biol Psychiatry 68, 197-204. doi: 10.1016/j.biopsych.2010.02.013.

Rashid, B., Damaraju, E., Pearlson, G. D. \& Calhoun, V. D., 2014. Dynamic connectivity states estimated from resting fMRI Identify differences among Schizophrenia, bipolar disorder, and healthy control subjects. Front Hum Neurosci 8, 897. doi: 10.3389/fnhum.2014.00897

Reed, J., McNamee, C., Rackstraw, S., Jenkins, J., Moss, D., 2004. Diglons are heterodimeric proteins composed of IgLON subunits, and diglon-CO inhibits neurite outgrowth from cerebellar granule cells. J Cell Sci 117, 3961-3973. doi: 10.1242/jcs.01261.

Roger, B., Al-Bassam, J., Dehmelt, L., Milligan, R.A., Halpain, S., 2004. MAP2c, but not tau, binds and bundles F-actin via its microtubule binding domain. Curr Biol 14(5), 363-371. doi: 10.1016/j.cub.2004.01.058.

Salzer, J.L., Rosen, C.L., Struyk, A.F., 1996. GPI-anchored proteins in neural cell adhesion. In: C DR, editor. Advences in Molecular and Cellular Biology. Cell adhesion Greenwich, CT, 193-222.

Sanz, R.L., Ferraro, G.B., Girouard, M.P., Fournier, A.E., 2017. Ectodomain shedding of Limbic System-Associated Membrane Protein (LSAMP) by ADAM Metallopeptidases promotes neurite outgrowth in DRG neurons. Sci Rep. 7(1), 7961. doi: 10.1038/s41598-01708315-0.

Schmidt, E. R., Brignani, S., Adolfs, Y., Lemstra, S., Demmers, J., Vidaki, M., Donahoo, A.L., Lilleväli, K., Vasar, E., Richards, L.J., et al., 2014. Subdomain-mediated axon-axon signaling and chemoattraction cooperate to regulate afferent innervation of the lateral habenula. Neuron 83(2), 372-387. doi: 10.1016/j.neuron.2014.05.036.

Sharma, K., Schmitt, S., Bergner, C.G., Tyanova, S., Kannaiyan, N., Manrique-Hoyos, N., Kongi, K., Cantuti, L., Hanisch, U.K., Philips, M.A., et al., 2015. Cell type- and brain region-resolved mouse brain proteome. Nat Neurosci 18, 1819-1831. doi: 10.1038/nn.4160.

Singh, K., Loreth, D., Pöttker, B., Hefti, K., Innos, J., Schwald, K., Hengstler, H., Menzel, L., Sommer, J. C., Radyushkin, K., Kretz, O., Philips, M. A., Haas, A. C., Frauenknecht, K., Lilleväli, K., Heimrich, B., Vasar, E., Schaefer, M. K. E., 2018. Neuronal growth and behavioral alterations in mice deficient for the psychiatric disease-associated Negr1 gene.Front. Mol. Neurosci. doi: 10.3389/fnmol.2018.00030.

Struyk, A. F., Canoll, P. D., Wolfgang, M. J., Rosen, C. L., D’Eustachio, P., Salzer, J. L., 
1995. Cloning of Ntm defines a new subfamily of differentially expressed neural cell adhesion molecules. J Neurosci 15, 2141-2156.

Tan, R. P. A., Leshchyns'ka, I., Sytnyk, V., 2017. Glycosylphosphatidylinositol- Anchored Immunoglobulin Superfamily Cell Adhesion Molecules and Their Role in Neuronal Development and Synapse Regulation. Front Mol Neurosci 10, 378. doi:10.3389/fnmol.2017.00378

Vanaveski, T., Singh, K., Narvik, J., Eskla, K-L., Visnapuu, T. , Heinla, I. , Jayaram, M., Innos, J., Lillevali , K., Philips , M-A., Vasar , E., 2017. Promoter-Specific Expression and Genomic Structure of IgLON Family Genes in mouse. Front Neurosci 11, 38. doi: 10.3389/fnins.2017.00038.

Yamada, M., Hashimoto, T., Hayashi, N., Higuchi, M., Murakami, A., Nakashima, T., Maekawa, S., Miyata, S., 2007. Synaptic adhesion molecule OBCAM; synaptogenesis and dynamic internalization. Brain Res 1165, 5-14. doi: 10.1016/j.brainres.2007.04.062.

Zacco, A., Cooper, V., Chantler, P. D., Fisher-Hyland, S., Horton, H.L., Levitt, P., 1990. Isolation, biochemical characterization and ultrastructural analysis of the limbic system associated membrane protein (LAMP), a protein expressed by neurons comprising functional neural circuits. J Neurosci 10, 73-90.

Zhang, X., Hu, X., Lei, H., Hu, J., Zhang, Y., 2016. Mechanical force-induced polymerization and depolymerization of F-actin at water/solid interfaces. Nanoscale 8(11), 6008-6013. doi: 10.1039/c5nr08713a. 
Figure legends

Figure 1. Lsamp and Ntm deficiency interferes with actin accumulation during the stage of neurite initiation. Confocal fluorescent images of DIV 0.25 hippocampal neurons derived from P0-1 Wt (A-D), $\mathrm{Lsamp}^{-/}$(E-H), $\mathrm{Ntm}^{-/}$(I-L), and $\mathrm{Lsamp}^{-/-} \mathrm{Ntm}^{-/}$mice (M-P). Phalloidin staining marks F-actin (red), MAP2 neurons (green), and DAPI stains nuclei (blue). Scale bar is $10 \mu \mathrm{m}$. Graph $(\mathbf{Q})$ represents quantification of F-actin rich accumulation area $\left(\mu \mathrm{m}^{2}\right)$ based on phalloidin intensity. $\mathrm{N}=70-75$ neurons evaluated per genotype from three independent experiments. Data represent mean $\pm \mathrm{SE},{ }^{*} \mathrm{p}<0.05$, ** $\mathrm{p}<0.01$, *** $\mathrm{p}<0.001$ by one-way analysis of variance (ANOVA) followed by Newman-Keuls multiple comparisons test.
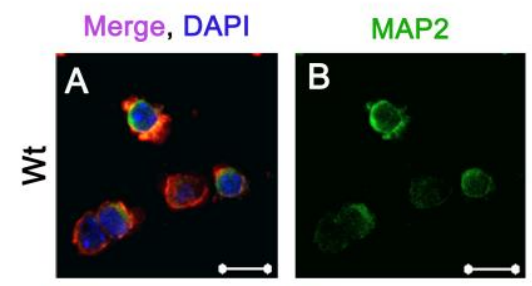

Phalloidin

Phalloidin
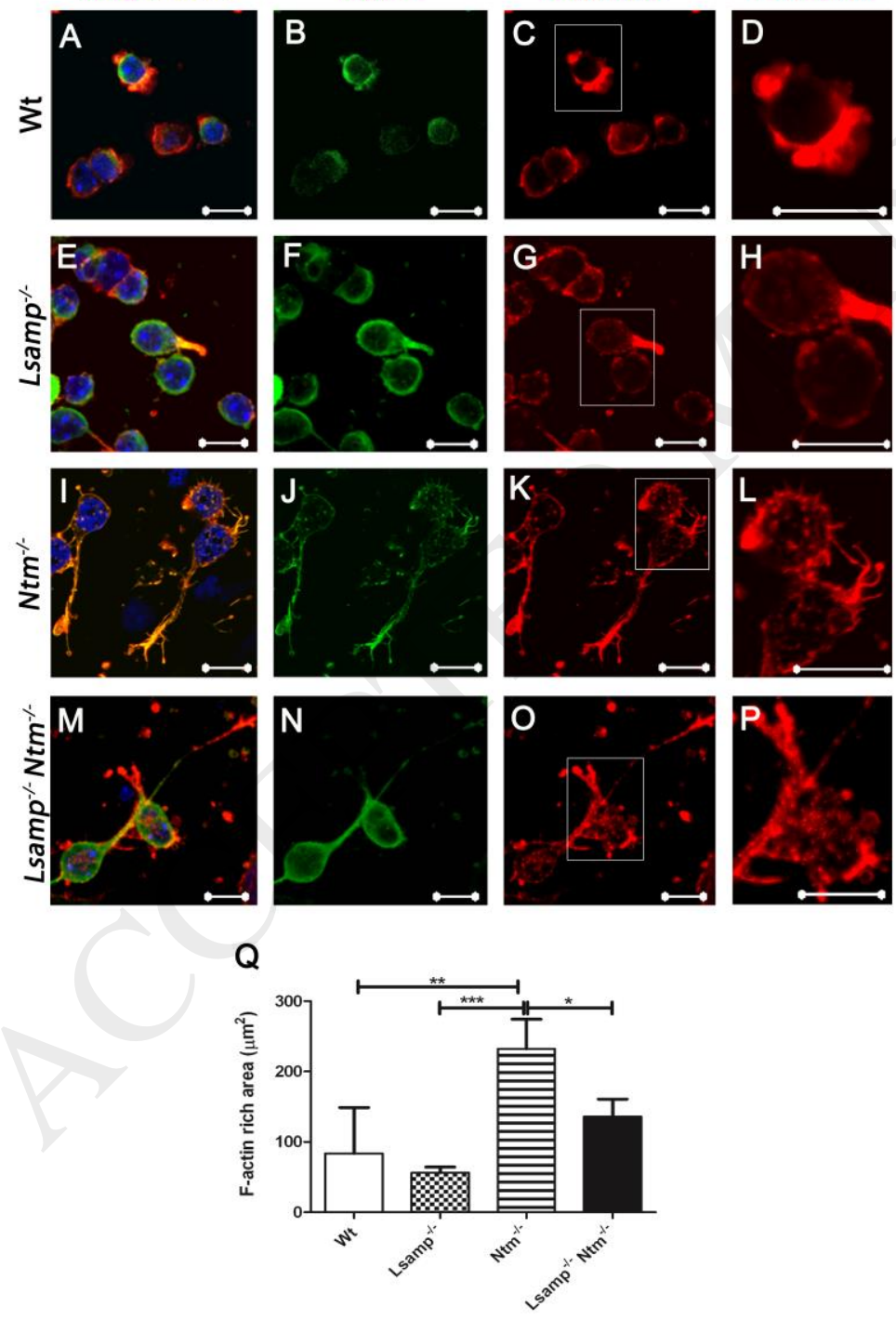
Figure 2. Ultrastructure of hippocampal neurons at the stage of neurite initiation. Scanning electron microscopy images of Wt $(\mathbf{A}, \mathbf{E}, \mathbf{I}), \operatorname{Lsamp}^{--}(\mathbf{B}, \mathbf{F}, \mathbf{J}), \mathrm{Ntm}^{--}(\mathbf{C}, \mathbf{G}, \mathbf{K})$, and $\mathrm{Lsamp}^{-/-\mathrm{Ntm}^{-/}}$(D, H, L) hippocampal neurons at DIV0.25. Arrows (E-H) point to a growing neurite; and arrows (I-L) to extending protrutions or filopodia on the neuronal surface. Scale bar in $\mathbf{A}-\mathbf{D}$ is $20 \mu \mathrm{m}$, in $\mathbf{E}-\mathbf{H}$ is $5 \mu \mathrm{m}$, in $\mathbf{I}-\mathbf{L}$ is $2 \mu \mathrm{m}$.
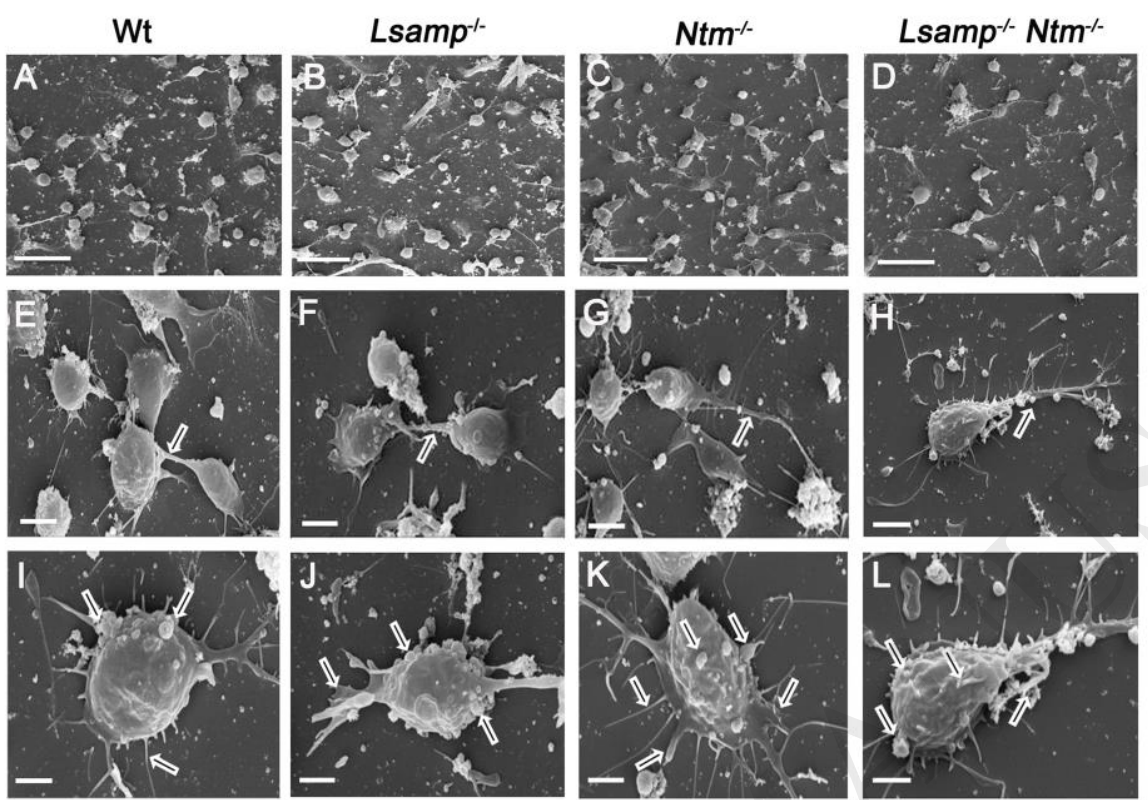

Figure 3. Morphometric analysis of hippocampal neurons in DIV3 dissociated culture. Representative images of pAAV-hSyn-RFP labelled hippocampal neurons derived from Wt (A), $\mathrm{Lsamp}^{-/}$(B), $\mathrm{Ntm}^{-/}$(C), and $\mathrm{Lsamp}^{-/-} \mathrm{Ntm}^{-/-}$(D) mice. Scale bar is $50 \mu \mathrm{m}$. Graphs (E-G) are obtained by neuron tracing Neuroleucida, and represent the number $(\mathbf{E})$, length $(\mathbf{F})$, and branch points (G) of the neurites per neuron. Data shown as mean $\pm \mathrm{SE}$ of $(\mathrm{N}=70-75)$ neurons of each genotype from three independent experiments. ${ }^{*} \mathrm{p}<0.05, * * \mathrm{p}<0.01$, $* * * \mathrm{p}<0.001$ by one-way analysis of variance (ANOVA) followed by Student-Newman-Keuls multiple comparisons test. 

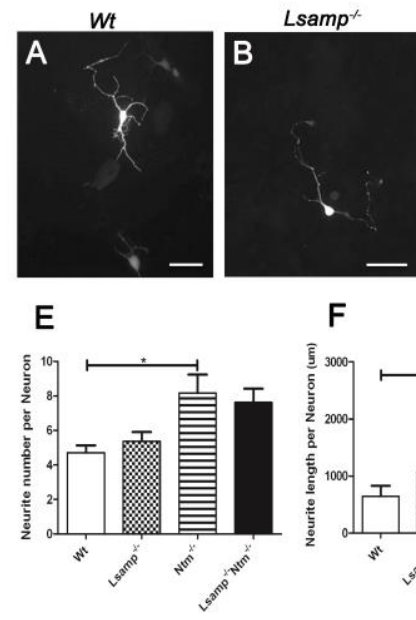

$\mathbf{F}$
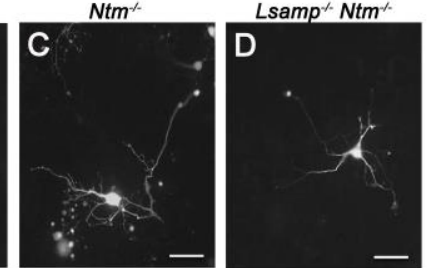

G
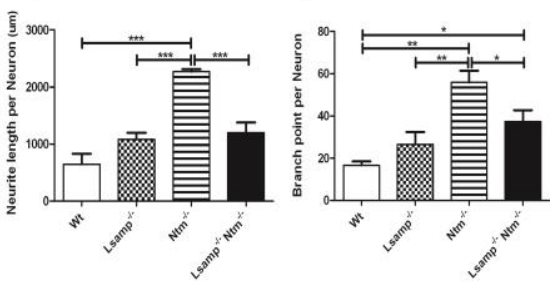

Figure 4. Effects of Lsamp and Ntm deficiencies on cellular proliferation. (A) High magnification image of PHH3 positive (green) primary cells at DIV3 undergoing mitosis. Nuclei are stained with DAPI (blue). Graph (B) displays the percentage of proliferative cells. Representative images of DIV3 hippocampal cultures derived from Wt $(\mathbf{C}-\mathbf{E}), \operatorname{Lsamp}^{-/}(\mathbf{F}-\mathbf{H})$, $\mathrm{Ntm}^{-/}(\mathbf{I}-\mathbf{K})$, and $\mathrm{Lsamp}^{-/-} \mathrm{Ntm}^{-/}(\mathbf{L}-\mathbf{N})$ mice show immunostainings with proliferation marker PHH3 (green) and neuronal marker, DCX (red). Scale bar in $\mathbf{A}$ is $10 \mu \mathrm{m}$ in $\mathbf{C}-\mathbf{N}$ is $100 \mu \mathrm{m}$. Data represent mean \pm SE from; $* \mathrm{p}<0.05, * * \mathrm{p}<0.01, * * * \mathrm{p}<0.001$ by one-way analysis of variance (ANOVA) followed by Newman-Keuls multiple comparisons test. 

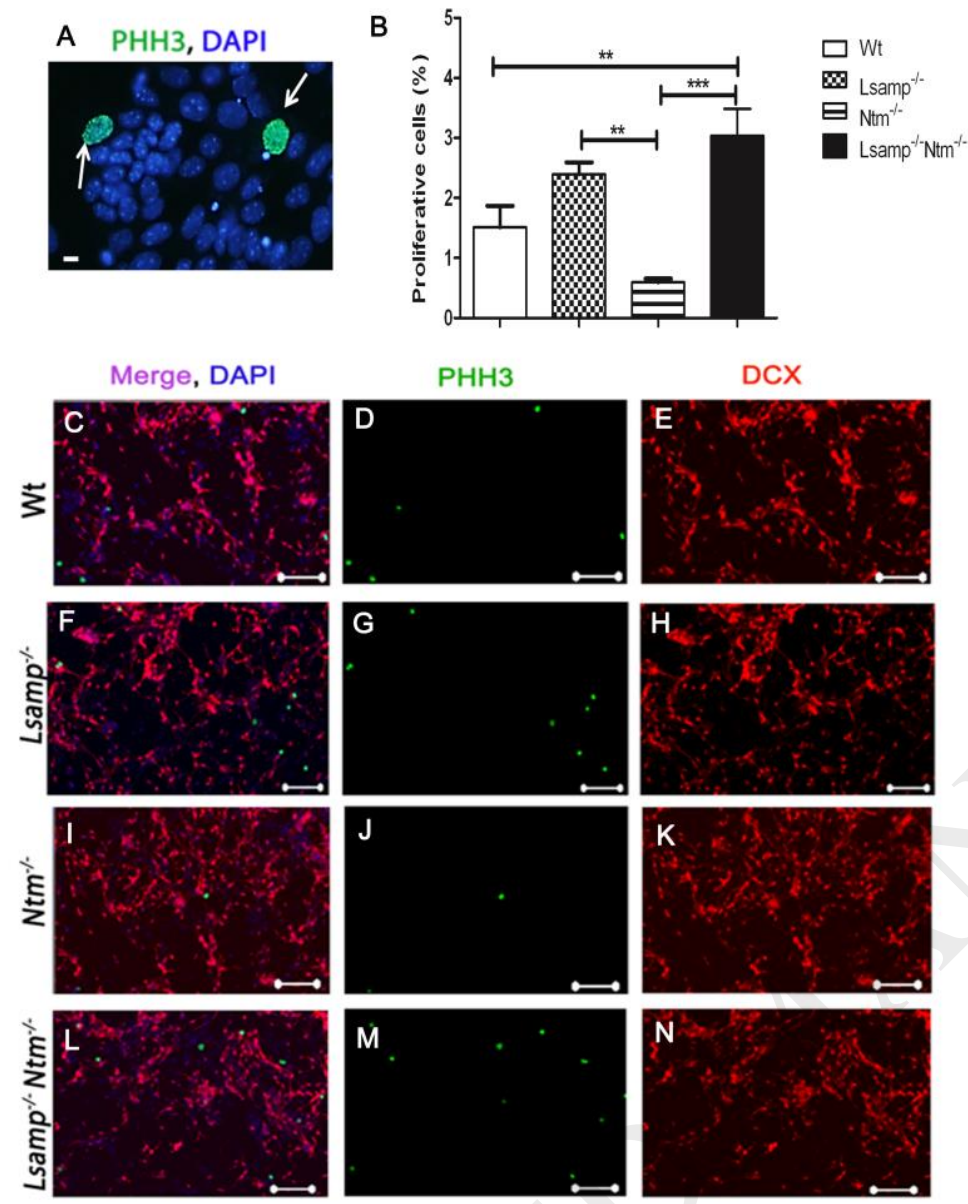

Figure 5. Lsamp deficiency decreases the percentage of apoptotic cells in hippocampal dissociated culture. Representative images showing dark brown TUNEL positive hippocampal neurons at DIV3 derived from Wt (A), $\mathrm{Lsamp}^{-/-}$(B), $\mathrm{Ntm}^{-/-}$(C), and $\mathrm{Lsamp}^{-/-} \mathrm{Ntm}^{-}$ /- (D) mice. Scale bar is $20 \mu \mathrm{m}$. Graph (E) displays the percentage of apoptotic cells among total number of cells. Data represent mean \pm SE from three independent experiments each with 10 independent image field evaluated per genotype; ***p $<0.001$ by one-way analysis of variance (ANOVA) followed by Newman-Keuls multiple comparisons test. 


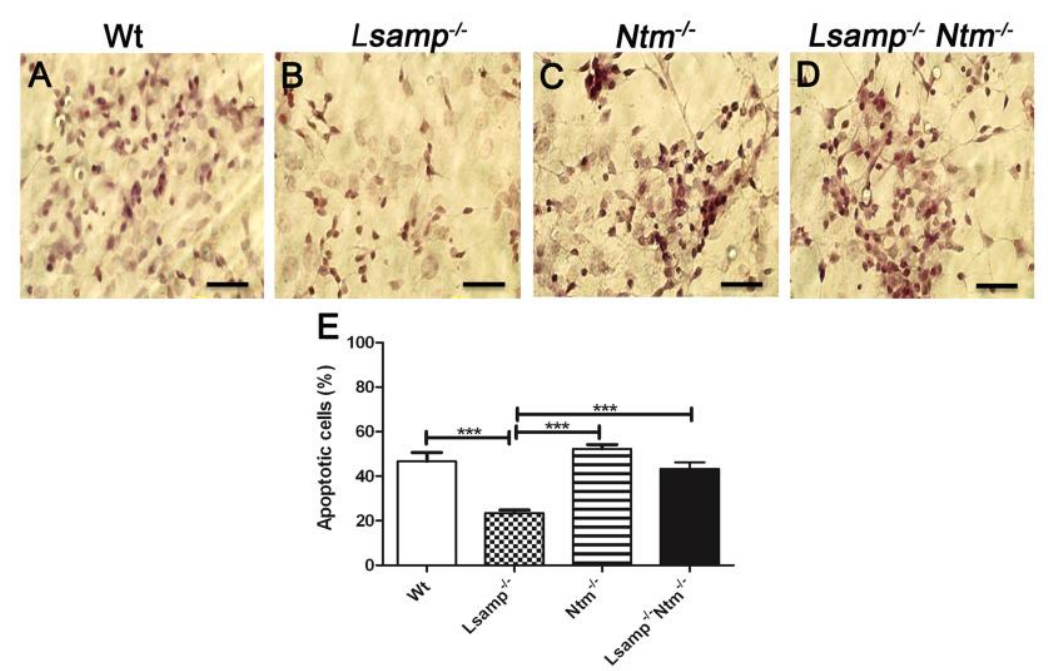

Figure 6. Histological analysis of IgLON/s deficient mice brain. Nissl-stained adult coronal brain sections of Wt (A), Lsamp ${ }^{-/}$(B), $\mathrm{Ntm}^{-/}$(C), and $\mathrm{Lsamp}^{-/} \mathrm{Ntm}^{-/}$(D) at the level of hippocampus; scale bar: $1.2 \mathrm{~mm}$. Immunostaining for neurofilament (NF) in saggital brain sections of Wt (E), samp $^{-/}(\mathbf{F}), \mathrm{Ntm}^{-/}(\mathbf{G})$, and $\mathrm{Lsamp}^{-/-} \mathrm{Ntm}^{-/}(\mathbf{H})$; scale bar: $1 \mathrm{~mm}$.
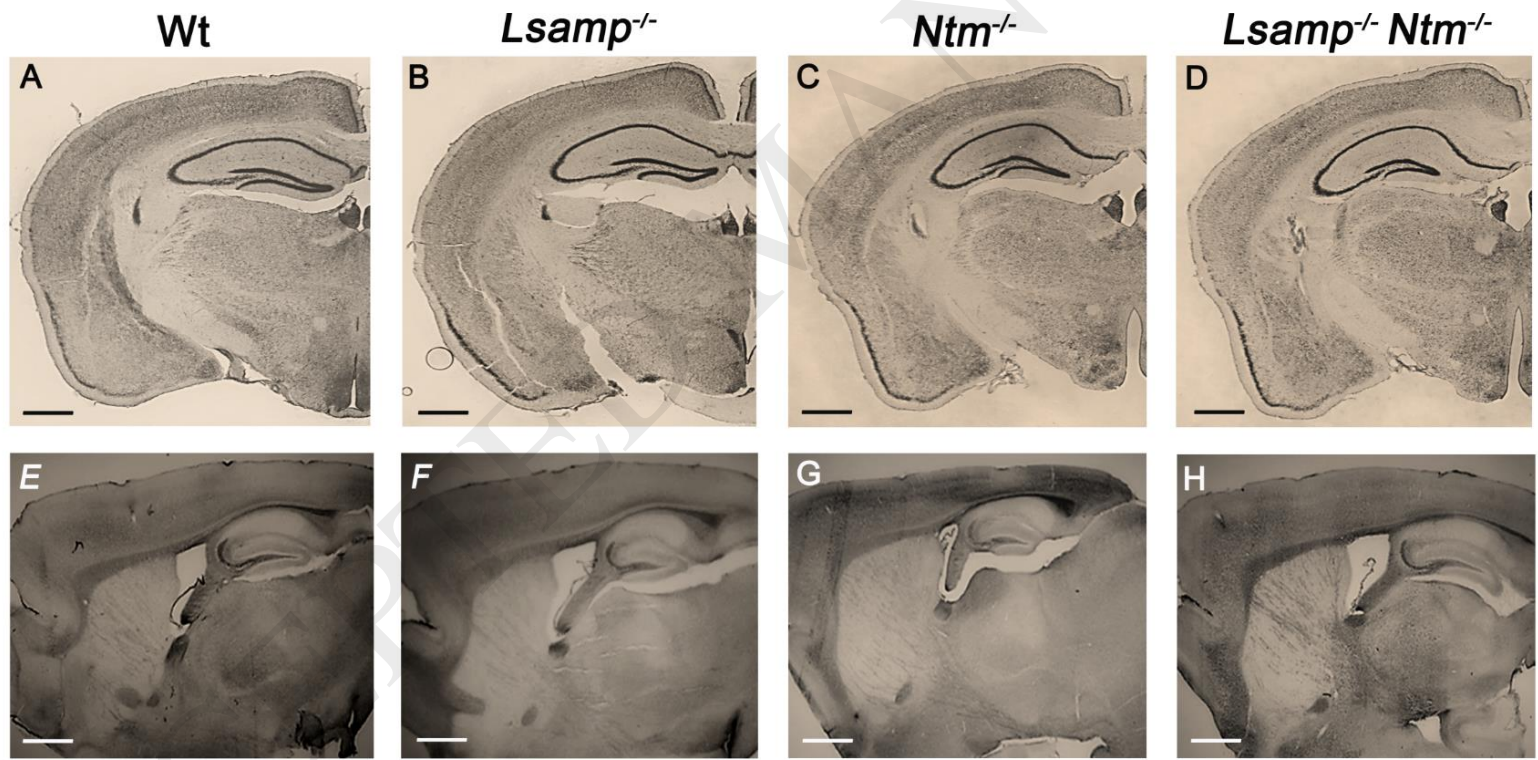
Figure 7. Behavioral analysis of $\mathrm{Lsamp}^{-/-}, \mathrm{Ntm}^{-/}$and $\mathrm{Lsamp}^{-/-} \mathrm{Ntm}^{-/-}$mice in motility box (A-C), Elevated plus-maze (D- F) and Morris water maze (G-J). Distance travelled (A), the number of rearings performed $(\mathbf{B})$, and distance travelled in the central part of the motility box (C) in the locomotor activity test in $\mathrm{Lsamp}^{-/-}$mice $(\mathrm{N}=11), \mathrm{Ntm}^{-/-}$mice $(\mathrm{N}=10), \mathrm{Lsamp}^{-/-} \mathrm{Ntm}^{-}$ / mice $(\mathrm{N}=10)$ and $\mathrm{Wt}$ mice $(\mathrm{N}=14)$. Closed arm entries (D), open arm entries (E), and protected head dips $(\mathbf{F})$ in the elevated plus maze in $\mathrm{Wt}(\mathrm{N}=11), \operatorname{Lsamp}^{-/-}(\mathrm{N}=11), \mathrm{Ntm}^{-/-}(\mathrm{N}=10)$ and Lsamp $^{-/-} \mathrm{Ntm}^{-/-}$mice $(\mathrm{N}=10)$. The learning curve $(\mathbf{G}, \mathbf{H})$ showing the time (in s) and distance (in $\mathrm{cm}$ ) to reach the submerged platform in Days 1-4. Average values per day, obtained by collapsing data of four trials for each animal, are presented in the Morris water maze. Time spent in the target quadrant (I) and swimming speed $(\mathrm{cm} / \mathrm{s})(\mathbf{J})$ in Lsamp $^{-/}$mice $(\mathrm{N}=10)$, Ntm $^{-/}$mice $(\mathrm{N}=10)$, Lsamp-/- Ntm-/- mice $(\mathrm{N}=10)$ and $\mathrm{Wt}$ mice $(\mathrm{N}=12)$ during Morris water maze. Data are presented as $\pm \mathrm{SE}\left({ }^{*} \mathrm{p}<0.05, * * \mathrm{p}<0.01, * * * \mathrm{p}<0.001\right.$. One-way ANOVA followed by Student-Newman-Keuls multiple comparisons test $),(\mathrm{cm}=$ centimetres; $\mathrm{m}=$ metres; $\mathrm{s}=$ seconds $)$. Dotted line in $(\mathbf{I})$ denotes chance level $(15 \mathrm{~s})$.

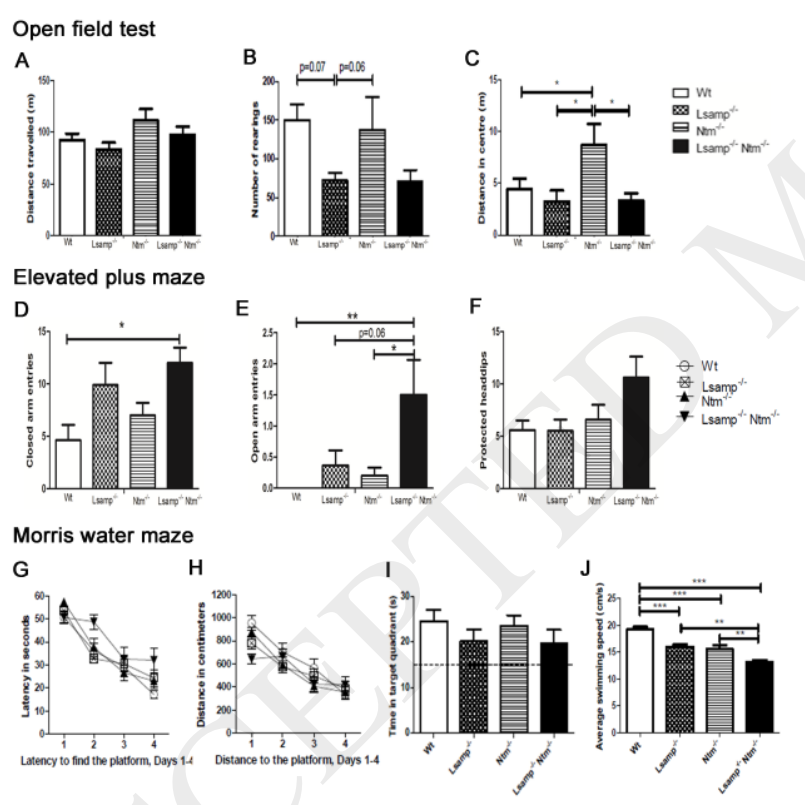

